\title{
A VARIATIONAL-HEMIVARIATIONAL INEQUALITY APPROACH TO THE LAMINATED PLATE THEORY UNDER SUBDIFFERENTIAL BOUNDARY CONDITIONS*
}

\author{
$\mathrm{BY}$ \\ P. D. PANAGIOTOPOULOS \\ (Aristotle University, Thessaloniki, Greece; Technical University of Aachen, Federal Republic of Germany) \\ AND \\ G. E. STAVROULAKIS (Aristotle University, Thessaloniki, Greece)
}

\begin{abstract}
In this paper the delamination problem for laminated plates is studied. A nonmonotone multivalued law is introduced in order to describe the interlaminar bonding forces. This law is written as the generalized gradient in the sense of F. H. Clarke of an appropriately defined nonconvex superpotential. Moreover, monotone boundary conditions of the subdifferential type are assumed to hold. The problem is formulated as a variational-hemivariational inequality expressing the principle of virtual work in inequality form. By using compactness and monotonicity arguments, the existence and the approximation of the solution of this inequality are investigated.
\end{abstract}

1. Introduction. In the present paper we deal with laminated plates, allowing for debonding of the laminae. The developed theory also holds for any type of layered plates or sandwich plates. The interaction between the laminae is described by a nonmonotone, possibly multivalued law connecting the interlaminar bonding forces with the corresponding relative displacements. This law permits the formulation of the problem as a hemivariational inequality (cf. [1]-[3]) if classical boundary conditions are assumed to hold. In the case of more complicated boundary conditions but of monotone type, which are derived through subdifferentiation from a convex superpotential [4], we obtain a variational-hemivariational inequality [5]. This variational-hemivariational inequality is studied here from the point of view of the existence and the approximation of his solution.

The considered problem is a unilateral one since its variational formulation is an inequality. Unilateral problems in the theory of plates were first studied (see, e.g., [6, $3]$ ) in the context of convexity and they give rise to variational inequalities. Here the nonmonotone interlaminar law is expressed in terms of a nonconvex superpotential [1] by forming its generalized gradient (see, e.g., [7]). Hemivariational inequalities

${ }^{*}$ Received July 30, 1986.

(C) 1988 Brown University 
in the framework of Kirchhoff and von Kármán plate theory, but for a single plate, have already been studied in $[8,9]$.

Due to the lack of monotonicity of the interlaminar law, we cannot use generally the monotonicity arguments widely applied in the theory of variational inequalities $[6,3]$; here compactness-average value arguments are applied, combined with the well-known monotonicity argument of the theory of variational inequalities. It is worth noting that these two arguments are completely independent, which might be useful in other areas, such as in the case of nonmonotone perturbations of multivalued equations involving monotone operators.

2. Derivation of the variational formulation of the problem. Let us consider a laminated plate consisting for the present of two laminae (Fig. 1a). Each lamina is an elastic plate and is referred to a right-handed orthogonal Cartesian coordinate system $O x_{1} x_{2} x_{3}$ (Fig. 1b). The two plates have constant thicknesses $h_{1}$ and $h_{2}$, and the middle surface of each plate coincides with the $O x_{1} x_{2}$-plane. Let $\Omega_{1}$ and $\Omega_{2}$ be two open, bounded, and connected subsets of $R^{2}$ and suppose that their boundaries $\Gamma_{1}$, $\Gamma_{2}$ are appropriately smooth (Lipschitzian boundaries $C^{0,1}$ are sufficient). $\Omega_{1}$ and $\Omega_{2}$ are occupied by the middle surfaces of the plates in their undeformed state. On $\Omega^{\prime} \subset \Omega_{1} \cap \Omega_{2}\left(\Omega^{\prime}\right.$ such that $\bar{\Omega}^{\prime} \cap \Gamma_{1}=\varnothing$ and $\left.\bar{\Omega}^{\prime} \cap \Gamma_{2}=\varnothing\right)$ the plates are bonded together in such a manner as to act as an integral structural element. We denote by $\zeta_{i}(x)$ the deflection of the point $x=\left\{x_{1}, x_{2}, x_{3}\right\}$ and by $f_{i}=\left(0,0, f_{3 i}\right), f_{3 i}=f_{3 i}(x)$ (hereafter called $f_{i}$ for simplicity) the distributed load of the $i$ th plate per unit area of the middle surface, where $i=1,2$ denotes the upper and the lower plate $\Omega_{1}, \Omega_{2}$, respectively (Fig. 1a).

The classical (Kirchhoff) theory of plates gives rise to the following system of differential equations:

$$
K_{i} \Delta \Delta \zeta_{i}=f_{i} \text { in } \Omega_{i}, i=1,2, \text { for the two plates. }
$$

Here $K_{i}=E_{i} h_{i}^{3} /\left(12\left(1-\nu_{i}^{2}\right)\right)$ is the bending rigidity of the $i$ th plate with $E_{i}$ and $\nu_{i}$ the modulus of elasticity and the Poisson ratio, respectively. We consider here for simplicity isotropic homogeneous plates. However, for further study we need only the bilinear form $\alpha\left(\zeta_{i}, z_{i}\right)$ of the elastic forces of each plate, and the whole study is independent of the specific form of $\alpha\left(\zeta_{i}, z_{i}\right)$ : only its coerciveness and its boundedness are needed. Therefore, we can replace $(2.1)$ by the respective equation for orthotropic or anisotropic, homogeneous or nonhomogeneous plates, as is often the case for laminated plates. It is well known (e.g., [10], p. 220) that the interlaminar normal stress $\sigma_{33}$ is responsible for the delamination effects in laminated, layered, or sandwich plates. Therefore, we split $f_{i}$ into $\overline{\bar{f}}_{i} \in L^{2}\left(\Omega_{i}\right)$, which is the given external loading acting on the $i$ th plate, and into $\bar{f}_{i}$, which describes the interaction between the two plates due to the binding material, i.e.,

$$
f_{i}=\overline{\bar{f}}_{i}+\bar{f}_{i} \quad \text { in } \Omega_{i}, \quad i=1,2 .
$$

To describe the bonding effect from the phenomenological point of view we can consider diagrams I, II, III of Fig. 2b, which take into consideration local cracking and crushing effects (cf. [1-3]) at the interface, characterized by brittle or semibrittle 

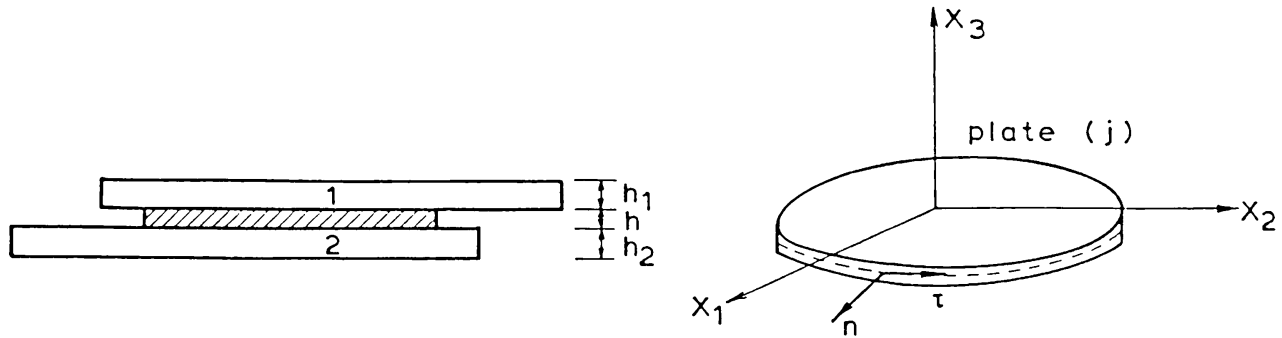

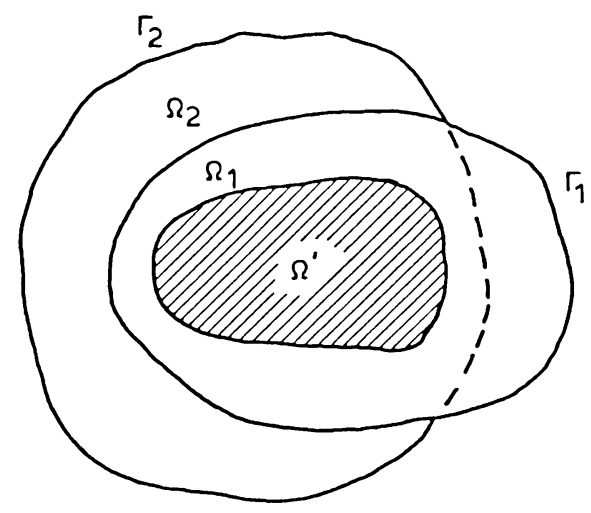

(a)

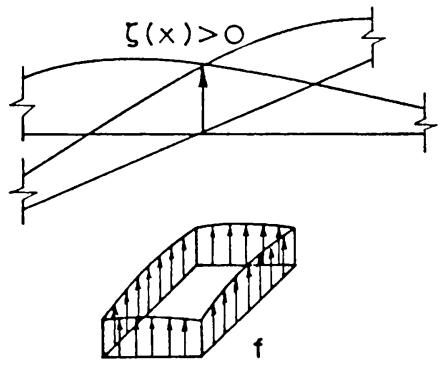

(b)

FIG. 1. On the geometry of the laminated plate

(dashed line) behavior (debonding effects). This graph corresponds to the adhesive contact of two laminae having negligible initial thickness of adhesive material between them. According to the assumption of plate theory, the two laminae are incompressible in the direction $O x_{3}$ and thus the condition of nonpenetrability should be described by a vertical branch $A B$. However, here, in order to take into account the possibility of elastic deformations of the laminae in the direction $O x_{3}$, we allow the line $A B$ to have a small slope (line $A B^{\prime}$ ). Analogously, the graph of Fig. 2c corresponds to the more realistic case of a bonding sheet between the two laminae of thickness $h \geq h^{\prime}$. It is worth noting that the developed theory here simulates the action of the adhesive material only in the direction normal to the plate. Interlayer slip, which together with the debonding normal to the laminae constitutes the main cause of the degradation of laminated plates, may occur in the framework of the theory presented here as a result of the Bernoulli assumption for each plate. A more complete study of the connection of debonding due to bending and that due to plane interlaminar stresses could be achieved only in the framework of von Kárman plate theory and will be attempted elsewhere. In the present paper we make the general assumption that $\bar{f}_{i}$ is generally a multivalued nonmonotone function (cf. Fig. 2d) of the relative displacement of two plates. 


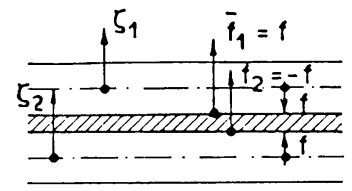

(a)

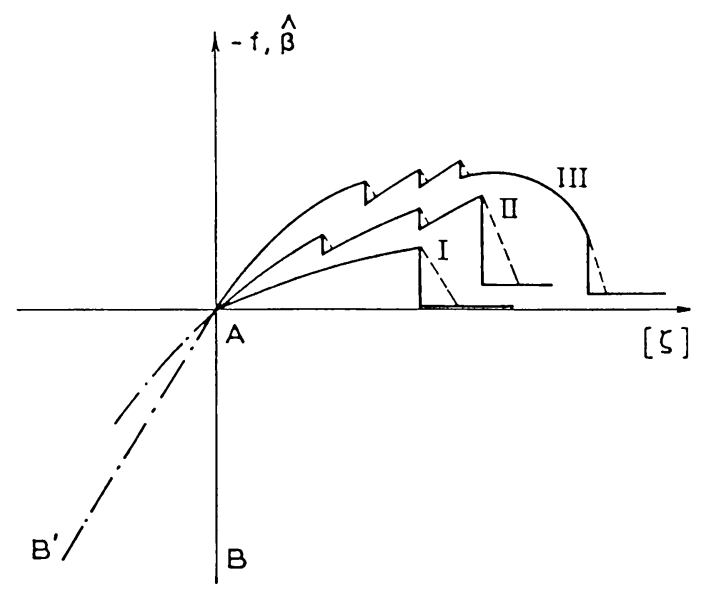

(b)
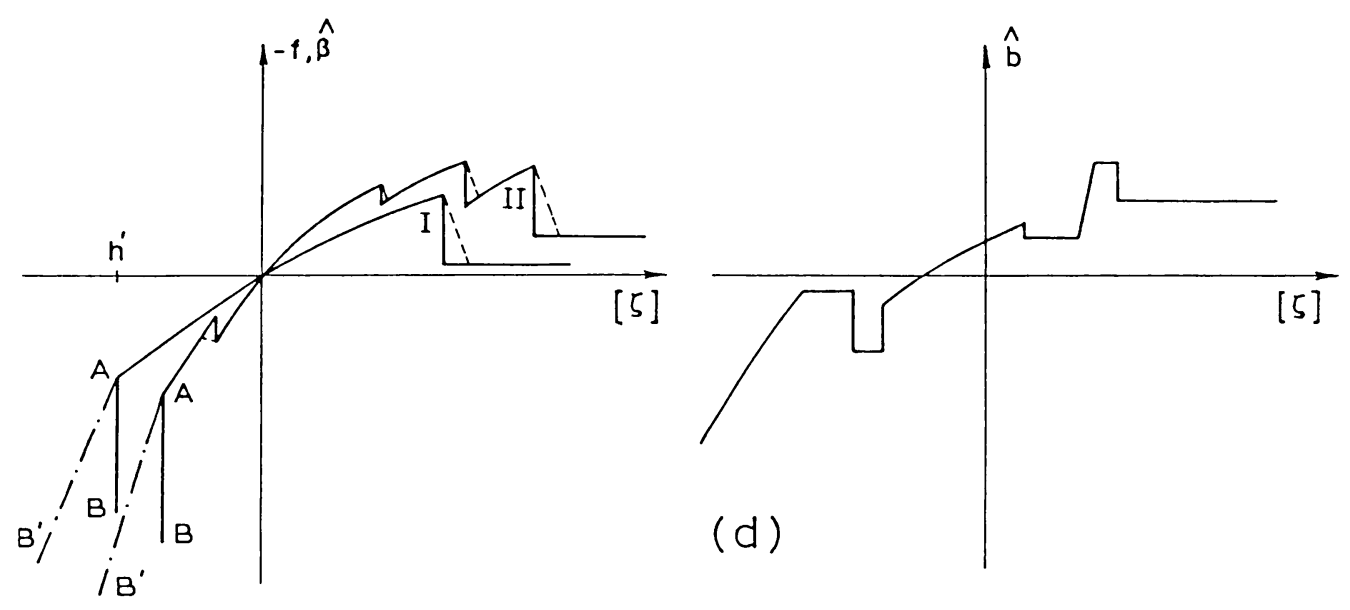

(c)

FIG. 2. Interlaminar force diagrams

$$
[\zeta]=\zeta_{1}-\zeta_{2} \quad \text { (see Fig. 2). }
$$

We write that

$$
-\bar{f}_{1} \in \hat{\beta}\left(\zeta_{1}-\zeta_{2}\right) \quad \text { on } \Omega^{\prime} \subset \Omega_{1} \cap \Omega_{2}
$$

(where $\bar{\Omega}^{\prime} \cap \Gamma_{1}=\varnothing$ ),

$$
+\bar{f}_{2} \in \hat{\beta}\left(\zeta_{1}-\zeta_{2}\right) \quad \text { on } \Omega^{\prime} \subset \Omega_{1} \cap \Omega_{2}
$$

(where $\bar{\Omega}^{\prime} \cap \Gamma_{2}=\varnothing$ ),

$$
\bar{f}_{1}=-\bar{f}_{2}=f
$$


and

$$
\begin{array}{ll}
\bar{f}_{1}=0 & \text { on } \Omega_{1}-\Omega^{\prime}, \\
\bar{f}_{2}=0 & \text { on } \Omega_{2}-\Omega^{\prime} .
\end{array}
$$

The graph of $\hat{\beta}$ may include vertical "filled in" gaps of finite length (Fig. 2b,c,d).

Further, we assume generally that $\hat{\beta}: R \rightarrow P(R)$ is a nonmonotone multivalued function, which is obtained in the following way. Let $\beta$ be a locally bounded, measurable function $\beta: R \rightarrow R$, i.e., $\beta \in L_{\text {loc }}^{\infty}(R)$. Moreover, for any $\varepsilon>0$ and $\xi \in R$ we define the numbers

$$
\bar{\beta}_{\varepsilon}\left(\xi_{1}\right)=\underset{\left|\xi_{1}-\xi\right|<\varepsilon}{\operatorname{ess} \sup } \beta(\xi)
$$

and

$$
\underline{\beta}_{\varepsilon}\left(\xi_{1}\right)=\underset{\left|\xi_{1}-\xi\right|<\varepsilon}{\operatorname{essinf}} \beta(\xi)
$$

which are increasing and decreasing functions of $\varepsilon$, respectively. So as $\varepsilon \rightarrow 0$ we may formulate $\lim \bar{\beta}_{\varepsilon}(\xi)=\bar{\beta}(\xi)$ and $\lim \underline{\beta}_{\varepsilon}(\xi)=\underline{\beta}(\xi)$, and we define the multivalued function $\hat{\beta}: R \rightarrow P(R)$ by setting

$$
\hat{\beta}(\xi)=[\underline{\beta}(\xi), \bar{\beta}(\xi)] .
$$

According to [11], if $\beta\left(\xi_{ \pm 0}\right)$ exists for every $\xi \in R$, then a locally Lipschitz function $j: R \rightarrow R$ can be determined such that

$$
\hat{\beta}(\xi)=\bar{\partial} j(\xi) .
$$

Here $j$ is defined up to an additive constant by the relation

$$
j(\xi)=\int_{0}^{\xi} \beta(t) d t
$$

and $\bar{\partial}$ denotes the generalized gradient of F. H. Clarke [7]. Thus we write (2.4a) in the form

$$
-\bar{f}_{1} \in \hat{\beta}(\zeta)=\bar{\partial} j(\zeta)
$$

which is equivalent by definition to the hemivariational inequality

$$
j^{0}(\xi, z-\xi) \geq-\bar{f}_{1}(z-\xi) \text { for all } z \in R .
$$

Here $j^{0}(\cdot, \cdot)$ denotes the directional derivative in the sense of F. H. Clarke defined by

$$
\begin{aligned}
j^{0}(\xi, z) & =\limsup _{\lambda \rightarrow 0_{+}, h \rightarrow 0} \frac{j(\xi+h+\lambda z)-j(\xi+h)}{\lambda} \\
& =\lim _{\lambda \rightarrow 0_{+}, h \rightarrow 0} \sup _{\lambda} \frac{1}{\lambda} \int_{\xi+h}^{\xi+h+\lambda z} \beta(\xi) d \xi .
\end{aligned}
$$

Note here that Eq. (2.11) may be interpreted physically as a nonconvex superpotential relation [1,3] describing the behavior of the adhesive material between the plates.

Now we can proceed to the derivation of the variational formulation of the problem. From (2.1), assuming sufficiently regular functions, we get the expressions

$$
\alpha\left(\zeta_{i}, z_{i}\right)=\int_{\Omega_{i}} f_{i} z_{i} d \Omega_{i}+\int_{\Gamma_{i}} \bar{Q}_{i}\left(\zeta_{i}\right) z_{i} d \Gamma_{i}-\int_{\Gamma_{i}} M_{i}\left(\zeta_{i}\right) \frac{\partial z_{i}}{\partial n_{i}} d \Gamma_{i}, \quad \forall z_{i} \in Z_{i}, \quad i=1,2
$$


Here $n_{i}$ denotes the outward unit normal vector to $\Gamma_{i}$,

$$
\alpha(\zeta, z)=K \int_{\Omega}\left[(1-\nu) \zeta_{, \alpha \beta} z_{, \alpha \beta}+\nu \Delta \zeta \Delta z\right] d \Omega, \quad \alpha, \beta=1,2,
$$

is the bilinear form of the elastic energy of each lamina,

$$
M(\zeta)=-K\left[\nu \Delta \zeta+(1-\nu)\left(2 n_{1} n_{2} \zeta_{, 12}+n_{1}^{2} \zeta_{, 11}+n_{2}^{2} \zeta_{, 22}\right)\right]
$$

is the bending moment and

$$
\bar{Q}(\zeta)=Q(\zeta)-\frac{\partial M(\zeta)}{\partial \tau}=-K\left[\frac{\partial \Delta \zeta}{\partial n}+(1-\nu) \frac{\partial}{\partial \tau}\left[n_{1} n_{2}\left(\zeta_{, 22}-\zeta_{.11}\right)+\left(n_{1}^{2}-n_{2}^{2}\right) \zeta_{.12}\right]\right]
$$

is the total shearing force on the plate boundary $\Gamma$. Equation (2.14) expresses the "principle of virtual work" for each lamina; $Z_{i}$ denotes the appropriate vector space for the deflections $\zeta_{i}$, which will be defined later. We write Eqs. (2.14) for $i=1,2$ and add. Then from (2.12) we get, by setting $[\zeta]=\zeta_{1}-\zeta_{2}$ and $[z]=z_{1}-z_{2}$, the hemivariational inequality

$$
\begin{aligned}
\alpha_{1}\left(\zeta_{1}, z_{1}-\zeta_{1}\right)+\alpha_{2}\left(\zeta_{2}, z_{2}-\zeta_{2}\right)+\int_{\Omega^{\prime}} j^{0}([\zeta],[z]-[\zeta]) d \Omega \\
\geq \int_{\Gamma_{1}} \bar{Q}_{1}\left(z_{1}-\zeta_{1}\right) d \Gamma+\int_{\Gamma_{2}} \bar{Q}_{2}\left(z_{2}-\zeta_{2}\right) d \Gamma-\int_{\Gamma_{1}} M_{1} \frac{\partial z_{1}-\partial \zeta_{1}}{\partial n} d \Gamma \\
\quad-\int_{\Gamma_{2}} M_{2} \frac{\partial z_{2}-\partial \zeta_{2}}{\partial n} d \Gamma+\int_{\Omega_{1}} \overline{\bar{f}}_{1}\left(z_{1}-\zeta_{1}\right) d \Omega \\
\quad+\int_{\Omega_{2}} \overline{\bar{f}}_{2}\left(z_{2}-\zeta_{2}\right) d \Omega, \quad \forall z_{1} \in Z_{1}, \forall z_{2} \in Z_{2} .
\end{aligned}
$$

Further, we specify the boundary conditions of each plate: let us assume that general monotone boundary conditions $[12,3]$ hold on the boundaries of the two plates $\Gamma_{1}, \Gamma_{2}$.

$$
\begin{array}{cl}
M_{1} \in \hat{b}_{1}\left(\frac{\partial \zeta_{1}}{\partial n}\right) & \text { on } \Gamma_{1}, \\
-\bar{Q}_{1} \in \hat{b}_{1}^{\prime}\left(\zeta_{1}\right) & \text { on } \Gamma_{1}, \\
M_{2} \in \hat{b}_{2}\left(\frac{\partial \zeta_{2}}{\partial n}\right) & \text { on } \Gamma_{2}, \\
-\bar{Q}_{2} \in \hat{b}_{2}^{\prime}\left(\zeta_{2}\right) & \text { on } \Gamma_{2},
\end{array}
$$

where $\hat{b}_{1}, \hat{b}_{1}^{\prime}, \hat{b}_{2}, \hat{b}_{2}^{\prime}$ are maximal monotone operators from $R$ into $P(R)$. Then convex, l.s.c., proper functionals $\varphi_{1}, \varphi_{1}^{\prime}, \varphi_{2}, \varphi_{2}^{\prime}$ (convex superpotentials [4]) can be determined such that

$$
\hat{b}_{i}\left(\frac{\partial \zeta_{i}}{\partial n}\right)=\partial \varphi_{i}\left(\frac{\partial \zeta_{i}}{\partial n}\right), \quad i=1,2
$$

and

$$
\hat{b}_{i}^{\prime}\left(\zeta_{i}\right)=\partial \varphi_{i}^{\prime}\left(\zeta_{i}\right), \quad i=1,2,
$$

where $\partial$ denotes the subdifferential [13]. These general subdifferential boundary conditions include all the classical (bilateral) and all the unilateral monotone boundary 
conditions [3, 6] (e.g., unilateral contact, friction, plastic hinge effects, prevented rotation effects). Concerning the appropriate choice of $\varphi_{i}$ and $\varphi_{i}^{\prime}$ for each specific boundary condition we refer to [3]. Further, the functionals

$$
\Phi_{i}\left(z_{i}\right)=\left\{\begin{array}{l}
\int_{\Gamma_{1}} \varphi_{i}\left(\frac{\partial z_{i}}{\partial n}\right) d \Gamma, \quad \text { if } \varphi_{i}\left(\frac{\partial z_{i}}{\partial n}\right) \in L^{1}\left(\Gamma_{i}\right), \\
\infty \text { otherwise, } i=1,2
\end{array}\right.
$$

and

$$
\Phi_{i}^{\prime}\left(z_{i}\right)=\left\{\begin{array}{l}
\int_{\Gamma_{i}} \varphi_{i}^{\prime}\left(z_{i}\right) d \Gamma, \quad \text { if } \varphi_{i}^{\prime}\left(z_{i}\right) \in L^{1}\left(\Gamma_{i}\right), \\
\infty \text { otherwise, } i=1,2,
\end{array}\right.
$$

are introduced. They are convex, l.s.c., and proper on $H^{2}\left(\Omega_{i}\right), i=1,2$. Thus we are led to the following problem:

Problem 1. Find $\zeta_{1} \in Z_{1}, \zeta_{2} \in Z_{2}$ such as to satisfy the variational-hemivariational inequality.

$$
\begin{aligned}
& \alpha_{1}\left(\zeta_{1}, z_{1}-\zeta_{1}\right)+\alpha_{2}\left(\zeta_{2}, z_{2}-\zeta_{2}\right) \\
& \quad+\int_{\Omega^{\prime}} j^{0}([\zeta],[z]-[\zeta]) d \Omega+\Phi_{1}^{\prime}\left(z_{1}\right)-\Phi_{1}^{\prime}\left(\zeta_{1}\right) \\
& \quad+\Phi_{2}^{\prime}\left(z_{2}\right)-\Phi_{2}^{\prime}\left(\zeta_{2}\right)+\Phi_{1}\left(z_{1}\right)-\Phi_{1}\left(\zeta_{1}\right)+\Phi_{2}\left(z_{2}\right)-\Phi_{2}\left(\zeta_{2}\right) \\
& \quad \geq \int_{\Omega_{1}} \overline{\bar{f}}_{1}\left(z_{1}-\zeta_{1}\right) d \Omega+\int_{\Omega_{2}} \overline{\bar{f}}_{2}\left(z_{2}-\zeta_{2}\right) d \Omega, \quad \forall z_{1} \in Z_{1}, \forall z_{2} \in Z_{2} .
\end{aligned}
$$

Equation (2.27) constitutes a variational-hemivariational inequality expressing the principle of virtual work in its inequality form.

Obviously, all combinations of classical boundary conditions with boundary conditions of monotone type resulting from convex superpotentials give rise to variationalhemivariational inequalities analogous to (2.27). Only modifications of the range of the integrals in (2.25) and (2.26) will be necessary. Further, we shall study (2.27) on the assumption that the boundary conditions guarantee the coerciveness of the bilinear forms; i.e., a "rigid lamina" displacement (polynomial of first degree in $x_{1}$ and $x_{2}$ ) is not permitted. Moreover, we include the classical boundary conditions in $Z_{i}$. For homogeneous boundary conditions $Z_{i}$ is a closed linear subspace of $H^{2}\left(\Omega_{i}\right)$, whereas for inhomogeneous boundary conditions $Z_{i}$ is a closed linear subvariety of $H^{2}\left(\Omega_{i}\right)$. In the latter case an appropriate translation transforms the boundary conditions into homogeneous ones. For simplicity we first study the following problem (problem 2). We assume that

$$
\zeta_{2}=0 \quad \text { and } \quad \partial \zeta_{2} / \partial n=0 \quad \text { on } \Gamma_{2}
$$

and that

$$
\zeta_{1}=0 \text { and (2.19) holds on } \Gamma_{1} \text {. }
$$

These boundary conditions guarantee the coerciveness of the bilinear forms. Then $Z_{1}=H^{2}\left(\Omega_{1}\right) \cap \dot{H}^{1}\left(\Omega_{1}\right)$ and $Z_{2}=\dot{H}^{2}\left(\Omega_{2}\right)$. 
Problem 2. Find $\zeta_{1} \in Z_{1}$ and $\zeta_{2} \in Z_{2}$ such as to satisfy the variational-hemivariational inequality

$$
\begin{aligned}
\alpha_{1}\left(\zeta_{1}, z_{1}-\zeta_{1}\right)+\alpha_{2}\left(\zeta_{2}, z_{2}-\zeta_{2}\right) & +\int_{\Omega^{\prime}} j^{0}([\zeta],[z]-[\zeta]) d \Omega+\Phi_{1}\left(z_{1}\right)-\Phi_{1}\left(\zeta_{1}\right) \\
& \geq\left(\overline{\bar{f}}_{1}, z_{1}-\zeta_{1}\right)+\left(\overline{\bar{f}}_{2}, z_{2}-\zeta_{2}\right), \quad \forall z_{1} \in Z_{1}, \quad \forall z_{2} \in Z_{2} .
\end{aligned}
$$

The above is a model problem: the method applied to its study remains the same if other more general boundary conditions are considered provided that the coerciveness of the bilinear forms is preserved. Such more general problems will be examined in the sequel.

3. Existence and approximation results; the differentiable case. We assume that for some $\xi \in R$

$$
\underset{(-\infty,-\xi)}{\operatorname{ess} \sup } \beta(\xi) \leq \underset{(+\xi,+\infty)}{\operatorname{essinf}} \beta(\xi)
$$

i.e., that $\beta$ "ultimately" increases. Obviously we can assume without loss of generality (appropriate translation of the coordinate axes) that

$$
\underset{(-\infty,-\xi)}{\operatorname{ess} \sup } \beta(\xi) \leq 0 \leq \underset{(+\xi,+\infty)}{\operatorname{essinf}} \beta(\xi) .
$$

Note that it is possible that $\beta( \pm \infty)= \pm \infty$. We shall distinguish two problems with respect to $\Phi_{1}$ : in the first case we assume that $\operatorname{grad} \Phi_{1}(\cdot)$ exists everywhere ("differentiable" case), while in the second case $\Phi_{1}$ is generally l.s.c., not everywhere differentiable, and may take values in $(-\infty,+\infty], \Phi \not \equiv \infty$ (the "nondifferentiable" case).

Let us denote by $\langle\cdot, \cdot\rangle$ the duality pairing between the spaces $Z_{1}$ and $Z_{1}^{\prime}$. Note that (2.30) is equivalent to

$$
\begin{aligned}
\alpha_{1}\left(\zeta_{1}, z_{1}-\zeta_{1}\right)+\alpha_{2}\left(\zeta_{2}, z_{2}-\zeta_{2}\right) & +\int_{\Omega^{\prime}} j^{0}([\zeta],[z]-[\zeta]) d \Omega+\left\langle\operatorname{grad} \Phi_{1}\left(\zeta_{1}\right), z_{1}-\zeta_{1}\right\rangle \\
& \geq\left(\overline{\bar{f}}_{1}, z_{1}-\zeta_{1}\right)+\left(\overline{\bar{f}}_{2}, z_{2}-\zeta_{2}\right), \quad \forall z_{1} \in Z_{1}, \quad \forall z_{2} \in Z_{2} .
\end{aligned}
$$

Here $(\cdot, \cdot)$ denotes the $L^{2}\left(\Omega_{i}\right)$-inner products. Indeed (2.30) implies (3.3), as it results easily by setting $z_{i}=\zeta_{i}+\lambda\left(w_{i}-\zeta_{i}\right), \lambda \in(0,1)$, and letting $\lambda \rightarrow 0_{+}$. Here we use the fact that $\xi \rightarrow j^{0}([\zeta], \xi)$ is positively homogeneous. It is obvious that (3.3) implies (2.30) because of the convexity of $\Phi$.

We consider the regularized problem $2_{\varepsilon}$, which is defined as follows. Let $p$ be a mollifier, i.e., $p \in C_{C}^{\infty}(-1,+1), p>0$, with $\int_{-\infty}^{+\infty} p(\xi) d \xi=1$ and let

$$
\beta_{\varepsilon}=p_{\varepsilon} * \beta, \quad \varepsilon>0 \text {, }
$$

where $p_{\varepsilon}(\xi)=(1 / \varepsilon) p(\xi / \varepsilon)$ and $*$ denotes the convolution product. Then problem $2_{\varepsilon}$ reads

Problem $2_{\varepsilon}$. Find $\zeta_{1 \varepsilon} \in Z_{1}$ and $\zeta_{2 \varepsilon} \in Z_{2}$ such as to satisfy the variational equality

$$
\begin{aligned}
\alpha_{1}\left(\zeta_{1 \varepsilon}, z_{1}\right) & +\alpha_{2}\left(\zeta_{2 \varepsilon}, z_{2}\right)+\left\langle\operatorname{grad} \Phi_{1}\left(\zeta_{1 \varepsilon}\right), z_{1}\right\rangle \\
& +\int_{\Omega^{\prime}} \beta_{\varepsilon}\left(\left[\zeta_{\varepsilon n}\right]\right)[z] d \Omega=\left(\overline{\bar{f}}_{1}, z_{1}\right)+\left(\overline{\bar{f}}_{2}, z_{2}\right), \quad \forall z_{1} \in Z_{1}, \forall z_{2} \in Z_{2} .
\end{aligned}
$$


We consider a Galerkin basis of $Z_{i}, i=1,2$, and we denote by $Z_{i n}$ the corresponding $n$-dimensional subspace of $Z_{i}$. Then the following finite-dimensional problem is formulated.

Problem $2_{\varepsilon n}$. Find $\zeta_{1 \varepsilon n} \in Z_{1 n}, \zeta_{2 \varepsilon n} \in Z_{2 n}$ such as to satisfy the variational equality

$$
\begin{aligned}
\alpha_{1}\left(\zeta_{1 \varepsilon n}, z_{1}\right) & +\alpha_{2}\left(\zeta_{2 \varepsilon n}, z_{2}\right)+\left\langle\operatorname{grad} \Phi_{1}\left(\zeta_{1 \varepsilon n}\right), z_{1}\right\rangle \\
& +\int_{\Omega^{\prime}} \beta_{\varepsilon}\left(\left[\zeta_{\varepsilon n}\right]\right)[z] d \Omega=\left(\overline{\bar{f}}_{1}, z_{1}\right)+\left(\overline{\bar{f}}_{2}, z_{2}\right), \quad \forall z_{1} \in Z_{1 n}, \forall z_{2} \in Z_{2 n}
\end{aligned}
$$

Besides assumption (3.1), we assume further that

$$
\operatorname{grad} \Phi_{1}(0)=0 \text {. }
$$

Proposition 3.1. Under assumptions (3.2) and (3.7) and for $\overline{\bar{f}}_{i} \in L^{2}\left(\Omega_{i}\right), i=1,2$, problem 2 has a solution.

Proof. We write (3.6) in the form

$$
\left\langle\Lambda\left(\tilde{\zeta}_{\varepsilon n}\right), \tilde{z}\right\rangle=0, \quad \text { for } \tilde{z}=\left(z_{1}, z_{2}\right), \forall z_{1} \in Z_{1 n}, \forall z_{2} \in Z_{2 n} .
$$

Because of (3.2) we may determine $\rho_{1}>0$ and $\rho_{2}>0$ such that $\beta_{\varepsilon}(\xi) \geq 0$ if $\xi>\rho_{1}$, $\beta_{\varepsilon}(\xi) \leq 0$ if $\xi<-\rho_{1}$, and $\left|\beta_{\varepsilon}(\xi)\right| \leq \rho_{2}$ if $|\xi| \leq \rho_{1}$, and we may write

$$
\begin{aligned}
\int_{\Omega^{\prime}} \beta_{\varepsilon}\left(\left[\zeta_{\varepsilon n}\right]\right)\left[\zeta_{\varepsilon n}\right] d \Omega= & \int_{\left|\left[\zeta_{\varepsilon n}\right]\right|>\rho_{1}} \beta_{\varepsilon}\left(\left[\zeta_{\varepsilon n}\right]\right)\left[\zeta_{\varepsilon n}\right] d \Omega \\
& +\int_{\left|\left[\zeta_{\varepsilon n}\right]\right| \leq \rho_{1}} \beta_{\varepsilon}\left(\left[\zeta_{\varepsilon n}\right]\right)\left[\zeta_{\varepsilon n}\right] d \Omega \\
\geq & 0-\rho_{1} \rho_{2} \operatorname{mes} \Omega^{\prime} .
\end{aligned}
$$

Because of the boundary conditions the bilinear forms are coercive, i.e.,

$$
\alpha_{i}\left(\zeta_{i}, \zeta_{i}\right) \geq c_{i}\left\|\zeta_{i}\right\|^{2}, \quad \forall \zeta_{i} \in Z_{i}, c_{i} \text { const. }>0, i=1,2 .
$$

Here $\|\cdot\|$ denotes the $H^{2}$-norm.

Because of the monotonicity of $\operatorname{grad} \Phi_{1}$ and assumption (3.7) we have

$$
\left\langle\operatorname{grad} \Phi_{1}\left(\zeta_{1 \varepsilon n}\right)-\operatorname{grad} \Phi_{1}(0), \zeta_{1 \varepsilon n}-0\right\rangle \geq 0, \quad \forall \zeta_{1 \varepsilon n} \in Z_{1 n} .
$$

From (3.9), (3.10), and (3.11) we find that

$$
\begin{aligned}
\left(\Lambda\left(\tilde{\zeta}_{\varepsilon n}\right), \tilde{\zeta}_{\varepsilon n}\right)= & \alpha_{1}\left(\zeta_{1 \varepsilon n}, \zeta_{1 \varepsilon n}\right)+\alpha_{2}\left(\zeta_{2 \varepsilon n}, \zeta_{2 \varepsilon n}\right) \\
& +\left\langle\operatorname{grad} \Phi_{1}\left(\zeta_{1 \varepsilon n}\right), \zeta_{1 \varepsilon n}\right\rangle+\int_{\Omega^{\prime}} \beta_{\varepsilon}\left(\left[\zeta_{\varepsilon n}\right]\right)\left[\zeta_{\varepsilon n}\right] d \Omega-\left(\overline{\bar{f}}_{1}, \zeta_{1 \varepsilon n}\right)-\left(\overline{\bar{f}}_{2}, \zeta_{2 \varepsilon n}\right) \\
\geq & c_{1}\left\|\zeta_{1 \varepsilon n}\right\|^{2}+c_{2}\left\|\zeta_{2 \varepsilon n}\right\|^{2} \\
& +0-\rho_{1} \rho_{2} \operatorname{mes} \Omega^{\prime}-c_{3}\left\|\zeta_{1 \varepsilon n}\right\|-c_{4}\left\|\zeta_{2 \varepsilon n}\right\|, \quad c_{1}, c_{2}, c_{3}, c_{4} \text { const. }>0 .
\end{aligned}
$$

By Brouwer's theorem (3.8) has a solution $\tilde{\zeta}_{\text {en }}$ with $\left\|\tilde{\zeta}_{\varepsilon n}\right\| \leq c$.

Further, we shall investigate the behavior of the solution $\tilde{\zeta}_{\varepsilon n}$ of the finite-dimensional problem $2_{\varepsilon n}$ as $\varepsilon \rightarrow 0$ and $n \rightarrow \infty$. Due to the fact that $\left\{\zeta_{i \varepsilon n}\right\}$ is bounded in $Z_{i}, i=1,2$, we may extract a subsequence, again denoted by $\left\{\zeta_{i \varepsilon n}\right\}$, such as to satisfy for $\varepsilon \rightarrow 0, n \rightarrow \infty$

$$
\zeta_{i \varepsilon n} \rightarrow \zeta_{1} \text { weakly in } Z_{i}, i=1,2
$$


But since the imbeddings $H^{2}\left(\Omega_{i}\right) \subset L^{2}\left(\Omega_{i}\right), i=1,2$, are compact, we obtain

$$
\zeta_{i \varepsilon n} \rightarrow \zeta_{i} \text { strongly in } L^{2}\left(\Omega_{i}\right), i=1,2,
$$

and accordingly

$$
\zeta_{i \varepsilon n} \rightarrow \zeta_{i} \text { a.e. in } \Omega_{i}, i=1,2 .
$$

Further, we show that, under the assumption (3.2), $\left\{\beta_{\varepsilon}\left(\left[\zeta_{\varepsilon n}\right]\right)\right\}$ is weakly precompact in $L^{1}\left(\Omega^{\prime}\right)$. By the Dunford-Pettis theorem ([14], p. 239) it suffices to show that for each $\mu>0$ a $\delta(\mu)>0$ can be determined such that for $\omega \subset \Omega^{\prime}$ with mes $\omega<\delta$

$$
\int_{\omega}\left|\beta_{\varepsilon}\left(\left[\zeta_{\varepsilon n}\right]\right)\right| d \Omega<\mu .
$$

The inequality (cf. [15])

$$
\xi_{0}\left|\beta_{\varepsilon}(\xi)\right| \leq\left|\beta_{\varepsilon}(\xi) \xi\right|+\xi_{0} \sup _{|\xi| \leq \xi_{0}}\left|\beta_{\varepsilon}(\xi)\right|
$$

implies that

$$
\int_{\omega}\left|\beta_{\varepsilon}\left(\left[\zeta_{\varepsilon n}\right]\right)\right| d \Omega \leq \frac{1}{\xi_{0}} \int_{\omega}\left|\beta_{\varepsilon}\left(\left[\zeta_{\varepsilon n}\right]\right)\left[\zeta_{\varepsilon n}\right]\right| d \Omega+\int_{\omega\left|\left[\zeta_{\varepsilon n}(x)\right]\right| \leq \xi_{0}}\left|\beta_{\varepsilon}\left(\left[\zeta_{\varepsilon n}\right]\right)\right| d \Omega
$$

But

$$
\begin{aligned}
\int_{\Omega^{\prime}}\left|\beta_{\varepsilon}\left(\left[\zeta_{\varepsilon n}\right]\right)\left[\zeta_{\varepsilon n}\right]\right| d \Omega^{\prime}= & \int_{\left|\left[\zeta_{\varepsilon n}(x)\right]\right|>\rho_{1}}\left|\beta_{\varepsilon}\left(\left[\zeta_{\varepsilon n}\right]\right)\left[\zeta_{\varepsilon n}\right]\right| d \Omega \\
& +\int_{\left|\left[\zeta_{\varepsilon n}(x)\right]\right| \leq \rho_{1}}\left|\beta_{\varepsilon}\left(\left[\zeta_{\varepsilon n}\right]\right)\left[\zeta_{\varepsilon n}\right]\right| d \Omega^{\prime} \\
= & \int_{\left|\left[\zeta_{\varepsilon n}(x)\right]\right|>\rho_{1}}|\cdots| d \Omega^{\prime}-\int_{\left|\left[\zeta_{\varepsilon n}(x)\right]\right| \leq \rho_{1}}|\cdots| d \Omega^{\prime} \\
& +2 \int_{\left|\left[\zeta_{\varepsilon n}(x)\right]\right| \leq \rho_{1}}|\cdots| d \Omega^{\prime} \leq \int_{\left|\left[\zeta_{\varepsilon n}(x)\right]\right|>\rho_{1}}|\cdots| d \Omega^{\prime} \\
& +\int_{\left|\left[\zeta_{\varepsilon n}(x)\right]\right| \leq \rho_{1}}|\cdots| d \Omega^{\prime}+2 \int_{\left|\left[\zeta_{\varepsilon n}(x)\right]\right| \leq \rho_{1}}|\cdots| d \Omega^{\prime} \\
= & \int_{\Omega^{\prime}} \beta_{\varepsilon}\left(\left[\zeta_{\varepsilon n}\right]\right)\left[\zeta_{\varepsilon n}\right] d \Omega^{\prime}+2 \int_{\left|\left[\zeta_{\varepsilon n}(x)\right]\right| \leq \rho_{1}}\left|\beta_{\varepsilon}\left(\left[\zeta_{\varepsilon n}\right]\right)\left[\zeta_{\varepsilon n}\right]\right| d \Omega \\
= & \left(\overline{\bar{f}}_{1}, \zeta_{1 \varepsilon n}\right)+\left(\overline{\bar{f}}_{2}, \zeta_{2 \varepsilon n}\right)-\alpha_{1}\left(\zeta_{1 \varepsilon n}, \zeta_{1 \varepsilon n}\right)-\alpha_{2}\left(\zeta_{2 \varepsilon n}, \zeta_{2 \varepsilon n}\right) \\
& -\left\langle\operatorname{grad} \Phi_{1}\left(\zeta_{1 \varepsilon n}\right), \zeta_{1 \varepsilon n}\right\rangle+2 \int_{\mid\left[\zeta_{\varepsilon n}(x)\right] \leq \rho_{1}}\left|\beta_{\varepsilon}\left(\left[\zeta_{\varepsilon n}\right]\right)\left[\zeta_{\varepsilon n}\right]\right| d \Omega^{\prime} \\
\leq & +2 \rho_{1} \rho_{2} \operatorname{mes} \Omega^{\prime}-\left\langle\operatorname{grad} \Phi_{1}\left(\zeta_{1 \varepsilon n}\right)-\operatorname{grad} \Phi_{1}(0), \zeta_{1 \varepsilon n}-0\right\rangle \\
\leq & +2 \rho_{1} \rho_{2} \operatorname{mes} \Omega^{\prime},
\end{aligned}
$$

where again (3.11) has been applied (see also (3.9)). Further, we use the relation

$$
\sup _{|\xi| \leq \xi_{0}}\left|\beta_{\varepsilon}(\xi)\right| \leq \operatorname{ess~sup}_{|\xi| \leq \xi_{0}+1}|\beta(\xi)| .
$$

We can choose $\xi_{0}$ such that for all $\varepsilon$ and $n$

$$
\frac{1}{\xi_{0}} \int_{\omega}\left|\beta_{\varepsilon}\left(\left[\zeta_{\varepsilon n}\right]\right)\left[\zeta_{\varepsilon n}\right]\right| d \Omega^{\prime} \leq \frac{1}{\xi_{0}}\left(c+2 \rho_{1} \rho_{2} \operatorname{mes} \Omega^{\prime}\right) \leq \frac{\mu}{2}
$$


and $\delta$ such that (cf. (3.20)) for mes $\omega<\delta$

$$
\underset{|\xi| \leq \xi_{0}+1}{\operatorname{ess} \sup }|\beta(\xi)| \leq \frac{\mu}{2 \delta}
$$

Then

$$
\begin{aligned}
\int_{\omega} \sup _{\left|\left[\zeta_{\text {en }}(x)\right]\right| \leq \xi_{0}}\left|\beta_{\varepsilon}\left(\left[\zeta_{\varepsilon n}\right]\right)\right| d \Omega & \leq \operatorname{esssup}_{\left|\left[\zeta_{\text {cn }}(x)\right]\right| \leq \xi_{0}+1}\left|\beta\left(\left[\zeta_{\varepsilon}\right]\right)\right| \text { mes } \omega \\
& \leq \frac{\mu}{2 \delta} \delta=\frac{\mu}{2} .
\end{aligned}
$$

Equations (3.18), (3.19), (3.21), and (3.22) imply (3.16), i.e., that $\beta_{\varepsilon}\left(\left[\zeta_{\varepsilon n}\right]\right)$ is weakly precompact in $L^{1}\left(\Omega^{\prime}\right)$. Thus, as $\varepsilon \rightarrow 0$ and $n \rightarrow \infty$, a subsequence again denoted by $\left\{\beta_{\varepsilon}\left[\zeta_{\varepsilon n}\right]\right\}$ can be determined such that

$$
\beta_{\varepsilon}\left(\left[\zeta_{\varepsilon n}\right]\right) \rightarrow \chi \quad \text { weakly in } L^{1}\left(\Omega^{\prime}\right)
$$

From (3.6) we get

$$
\left|\left\langle\operatorname{grad} \Phi_{1}\left(\zeta_{1 \varepsilon n}\right), z_{1}\right\rangle\right| \leq c\left\|z_{1}\right\| z_{1}, \quad \forall z_{1} \in Z_{1}
$$

which implies that

$$
\left\|\operatorname{grad} \Phi_{1}\left(\zeta_{1 \varepsilon n}\right)\right\|_{Z_{1}^{\prime}} \leq c
$$

Thus we can determine a subsequence again denoted by $\left\{\operatorname{grad} \Phi_{1}\left(\zeta_{1 \varepsilon n}\right)\right\}$ such that as $\varepsilon \rightarrow 0, n \rightarrow \infty$

$$
\operatorname{grad} \Phi_{1}\left(\zeta_{1 \varepsilon n}\right) \rightarrow \Psi \text { weakly in } Z_{1}^{\prime}
$$

Thus by passing to the limit $n \rightarrow \infty, \varepsilon \rightarrow 0$ we get from (3.6) (note that $[z] \in$ $H^{2}\left(\Omega^{\prime}\right) \subset L^{\infty}\left(\Omega^{\prime}\right)$ for $\left.\Omega^{\prime} \subset R^{2}\right)$

$$
\begin{aligned}
\alpha_{1}\left(\zeta_{1}, z_{1}\right)+\alpha_{2}\left(\zeta_{2}, z_{2}\right)+\left\langle\Psi, z_{1}\right\rangle & +\int_{\Omega^{\prime}} \chi[z] d \Omega \\
& =\left(\overline{\bar{f}}_{1}, z_{1}\right)+\left(\overline{\bar{f}}_{2}, z_{2}\right), \quad \forall z_{1} \in Z_{1}, \forall z_{2} \in Z_{2} .
\end{aligned}
$$

The proof will be complete if we show that

$$
\Psi=\operatorname{grad} \Phi_{1}\left(\zeta_{1}\right) \text { in } Z_{1}^{\prime}
$$

and that

$$
\chi \in \hat{\beta}([\zeta]) \text { a.e. on } \Omega^{\prime} \text {. }
$$

To prove (3.29) we proceed as follows. We form the nonnegative-because of the monotonicity-expression

$$
X_{n}=\left\langle\operatorname{grad} \Phi_{1}\left(\zeta_{1 \varepsilon n}\right)-\operatorname{grad} \Phi_{1}(\theta), \zeta_{1 \varepsilon n}-\theta\right\rangle \geq 0, \quad \forall \theta \in Z_{1},
$$

which by means of $(3.6)$ becomes

$$
\begin{aligned}
X_{n}= & -\alpha_{1}\left(\zeta_{1 \varepsilon n}, \zeta_{1 \varepsilon n}\right)-\alpha_{2}\left(\zeta_{2 \varepsilon n}, \zeta_{2 \varepsilon n}\right) \\
& -\int_{\Omega^{\prime}} \beta_{\varepsilon}\left(\left[\zeta_{\varepsilon n}\right]\right)\left[\zeta_{\varepsilon n}\right] d \Omega+\left(\overline{\bar{f}}_{1}, \zeta_{1 \varepsilon n}\right)+\left(\overline{\bar{f}}_{2}, \zeta_{2 \varepsilon n}\right) \\
& -\left\langle\operatorname{grad} \Phi_{1}\left(\zeta_{1 \varepsilon n}\right), \theta\right\rangle-\left\langle\operatorname{grad} \Phi_{1}(\theta), \zeta_{1 \varepsilon n}-\theta\right\rangle \geq 0, \quad \forall \theta \in Z_{1} .
\end{aligned}
$$


We assume at present (the proof will be given in Lemma 3.1 separately) that

$$
\lim _{\substack{\varepsilon \rightarrow 0 \\ n \rightarrow \infty}} \int_{\Omega^{\prime}} \beta_{\varepsilon}\left(\left[\zeta_{\varepsilon n}\right]\right)\left[\zeta_{\varepsilon n}\right] d \Omega=\int_{\Omega^{\prime}} \chi[\zeta] d \Omega .
$$

Then (3.32) gives as $\varepsilon \rightarrow 0, n \rightarrow \infty$

$$
\begin{aligned}
0 \leq \lim \sup & X_{n} \leq-\alpha_{1}\left(\zeta_{1}, \zeta_{1}\right)-\alpha_{2}\left(\zeta_{2}, \zeta_{2}\right) \\
& \quad-\int_{\Omega^{\prime}} \chi[\zeta] d \Omega+\left(\overline{\bar{f}}_{1}, \zeta_{1}\right)+\left(\overline{\bar{f}}_{2}, \zeta_{2}\right)-\langle\Psi, \theta\rangle-\left\langle\operatorname{grad} \Phi_{1}(\theta), \zeta_{1}-\theta\right\rangle .
\end{aligned}
$$

From (3.28) and (3.34) we get

$$
\left\langle\Psi, \zeta_{1}-\theta\right\rangle-\left\langle\operatorname{grad} \Phi_{1}(\theta), \zeta_{1}-\theta\right\rangle \geq 0, \quad \forall \theta \in Z_{1},
$$

which implies by the well-known monotonicity argument or Minty's method (see, e.g., [6], p. 55) that

$$
\Psi=\operatorname{grad} \Phi_{1}\left(\zeta_{1}\right) \text { in } Z_{1}^{\prime} .
$$

It remains for us now to show that

$$
\chi \in \hat{\beta}([\zeta]) \text { a.e. in } \Omega^{\prime} .
$$

From (3.15) by applying Egoroff's theorem we can find that for every $\alpha>0, \omega_{1}$ and $\omega_{2}$ with mes $\omega_{1}<\alpha$ and mes $\omega_{2}<\alpha$ can be determined such that

$$
\zeta_{i \varepsilon n} \rightarrow \zeta_{i} \quad \text { uniformly in } \Omega_{i}-\omega_{i}, \quad i=1,2,
$$

where $\zeta_{i} \in L^{\infty}\left(\Omega_{i}-\omega_{i}\right)$. (Actually $\zeta_{i \varepsilon n} \rightarrow \zeta_{i}$ strongly in $L^{\infty}\left(\Omega_{i}\right)$ because of the compact imbedding $H^{2}\left(\Omega_{i}\right) \subset C^{0}\left(\bar{\Omega}_{i}\right)$ and the imbedding $C^{0}\left(\bar{\Omega}_{i}\right) \subset L^{\infty}\left(\Omega_{i}\right)$.) Because of the uniform convergence for any $\rho>0$ we can determine $\varepsilon_{0}<\rho / 2, n_{0}>2 / \rho$ such that for $\varepsilon<\varepsilon_{0}, n>n_{0}$

$$
\left|\zeta_{i \varepsilon n}-\zeta_{i}\right|<\rho / 2, \quad \forall x \in \Omega_{i}-\omega_{i}, \quad i=1,2 .
$$

Therefore for every $\alpha>0$ we can determine $\omega$ with mes $\omega<\alpha$ such that for any $\mu>0$ and for $\varepsilon<\varepsilon_{0}<\mu / 2$ and $n>n_{0}>2 / \mu$

$$
\left|\left[\zeta_{\varepsilon n}\right]-[\zeta]\right|<\mu / 2, \quad \forall x \in \Omega^{\prime}-\omega .
$$

We can easily verify that

$$
\beta_{\varepsilon}(\xi)=\left(p_{\varepsilon} * \beta\right)(\xi)=\int_{-\varepsilon}^{+\varepsilon} \beta(\xi-t) p_{\varepsilon}(t) d t \leq \underset{|t|<\varepsilon}{\operatorname{ess} \sup } \beta(\xi-t)
$$

and analogously

$$
\underset{|t|<\varepsilon}{\operatorname{ess} \inf } \beta(\xi-t) \leq \beta_{\varepsilon}(\xi)
$$

Thus from (3.40) (see also (2.7))

$$
\begin{aligned}
\beta_{\varepsilon}\left(\left[\zeta_{\varepsilon n}\right]\right) & \leq \underset{\left|\left[\zeta_{\zeta_{n}}\right]-\xi\right|<\varepsilon}{\operatorname{ess} \sup \beta(\xi)} \\
& \leq \underset{\left|\left[\zeta_{c_{n}}\right]-\xi\right|<\mu / 2}{\operatorname{ess} \sup } \beta(\xi) \leq \underset{|[\zeta]-\xi|<\mu}{\operatorname{ess} \sup } \beta(\xi)=\bar{\beta}_{\mu}([\zeta]) .
\end{aligned}
$$


Analogously,

$$
\underline{\beta}_{\mu}([\zeta]) \leq \operatorname{essinf}_{\mid \zeta \zeta]-\xi \mid<\mu} \beta(\xi) \leq \beta_{\varepsilon}\left(\left[\zeta_{\varepsilon n}\right]\right) .
$$

Choosing $e \geq 0$ a.e. in $\Omega^{\prime}-\omega$ with $e \in L^{\infty}\left(\Omega^{\prime}-\omega\right)$, we obtain from (3.42)

$$
\int_{\Omega^{\prime}-\omega} \underline{\beta}_{\mu}([\zeta]) e d \Omega \leq \int_{\Omega^{\prime}-\omega} \beta_{\varepsilon}\left(\left[\zeta_{\varepsilon n}\right]\right) e d \Omega \leq \int_{\Omega^{\prime}-\omega} \bar{\beta}_{\mu}([\zeta]) e d \Omega,
$$

and taking the limits $\varepsilon \rightarrow 0$ and $n \rightarrow \infty$ we find

$$
\int_{\Omega^{\prime}-\omega} \underline{\beta}_{\mu}([\zeta]) e d \Omega \leq \int_{\Omega^{\prime}-\omega} \chi e d \Omega \leq \int_{\Omega^{\prime}-\omega} \bar{\beta}_{\mu}([\zeta]) e d \Omega .
$$

Lebesgue's theorem implies as $\mu \rightarrow 0$ that

$$
\int_{\Omega^{\prime}-\omega} \underline{\beta}([\zeta]) e d \Omega \leq \int_{\Omega^{\prime}-\omega} \chi e d \Omega \leq \int_{\Omega^{\prime}-\omega} \bar{\beta}([\zeta]) e d \Omega .
$$

Since $e \geq 0$ is arbitrary, (3.45) implies that

$$
\chi \in \hat{\beta}([\zeta]) \text { a.e. in } \Omega^{\prime}-\omega
$$

and by taking $\alpha$ as small as we wish we obtain (3.37).

Lemma 3.1. As $\varepsilon \rightarrow 0, n \rightarrow \infty,(3.33)$ holds.

Proof. We form the difference

$$
\begin{aligned}
\int_{\Omega^{\prime}}\left\{\beta_{\varepsilon}\left(\left[\zeta_{\varepsilon n}\right]\right)\left[\zeta_{\varepsilon n}\right]-\chi[\zeta]\right\} d \Omega= & \int_{\Omega^{\prime}} \beta_{\varepsilon}\left(\left[\zeta_{\varepsilon n}\right]\right)\left(\left[\zeta_{\varepsilon n}\right]-[\zeta]\right) d \Omega \\
& +\int_{\Omega^{\prime}}[\zeta]\left(\beta_{\varepsilon}\left(\left[\zeta_{\varepsilon n}\right]\right)-\chi\right) d \Omega=A+B
\end{aligned}
$$

Since $H^{2}\left(\Omega^{\prime}\right)$ is imbedded into $L^{\infty}\left(\Omega^{\prime}\right),(3.24)$ implies that $\lim B=0$. This imbedding is also compact. On the other hand, (3.24) implies that

$$
\left\|\beta_{\varepsilon}\left(\left[\zeta_{\varepsilon}\right]\right)\right\|_{L^{\prime}\left(\Omega^{\prime}\right)}<c .
$$

Therefore

$$
|A| \leq\left\|\beta_{\varepsilon}\left(\left[\zeta_{\varepsilon n}\right]\right)\right\|_{L^{\prime}(\Omega)}\left\|\left[\zeta_{\varepsilon n}\right]-[\zeta]\right\|_{L^{\infty}\left(\Omega^{\prime}\right)}
$$

which yields that $\lim A=0$.

4. Study of the nondifferentiable case. In this case $\operatorname{grad} \Phi_{1}$ does not exist everywhere. In order to regularize the convex superpotential $\Phi$ we assume that a sequence of convex Gâteaux-differentiable functionals $\left\{\Phi_{1 \rho}\right\}$ exists, depending on a parameter $\rho$, with the properties:

(i) As $\rho \rightarrow 0, \Phi_{1 \rho}(z) \rightarrow \Phi_{1}(z), \forall z \in Z_{1}$,

(ii) $\operatorname{grad} \Phi_{1 \rho}(0)=0$, and

(iii) if $z_{\rho} \rightarrow z$ weakly in $Z_{1}$ for $\rho \rightarrow 0$, and if $\Phi_{1 \rho}\left(z_{\rho}\right)<M$, where $M$ is a constant, then

$$
\liminf _{\rho \rightarrow 0} \Phi_{1 \rho}\left(z_{\rho}\right) \geq \Phi(z)
$$

We now define the following regularized problem. 
Problem $2_{\varepsilon \rho}^{\prime}$. Find $\zeta_{i \varepsilon \rho} \in Z_{i}, i=1,2$, such as to satisfy the variational equality

$$
\begin{aligned}
\alpha_{1}\left(\zeta_{1 \varepsilon \rho}, z_{1}\right)+ & \alpha_{2}\left(\zeta_{2 \varepsilon \rho}, z_{2}\right)+\int_{\Omega^{\prime}} \beta_{\varepsilon}\left(\left[\zeta_{\varepsilon \rho}\right]\right)[z] d \Omega \\
& +\left\langle\operatorname{grad} \Phi_{\rho}\left(\zeta_{1 \varepsilon \rho}\right), z_{1}\right\rangle=\left(\overline{\bar{f}}_{1}, z_{1}\right)+\left(\overline{\bar{f}}_{2}, z_{2}\right), \quad \forall z_{i} \in Z_{i}, \quad i=1,2 .
\end{aligned}
$$

The corresponding discretized problem reads

Problem $2_{\varepsilon \rho n}^{\prime}$. Find $\zeta_{i \varepsilon \rho n} \in Z_{i n}, i=1,2$, such as to satisfy the variational equality

$$
\begin{aligned}
\alpha_{1}\left(\zeta_{1 \varepsilon \rho n}, z_{1}\right) & +\alpha_{2}\left(\zeta_{2 \varepsilon \rho n}, z_{2}\right)+\int_{\Omega^{\prime}} \beta_{\varepsilon}\left(\left[\zeta_{\varepsilon \rho n}\right]\right)[z] d \Omega \\
& +\left\langle\operatorname{grad} \Phi_{1 \rho}\left(\zeta_{1 \varepsilon \rho n}\right), z_{1}\right\rangle=\left(\overline{\bar{f}}_{1}, z_{1}\right)+\left(\overline{\bar{f}}_{2}, z_{2}\right), \quad \forall z_{i} \in Z_{i n}, \quad i=1,2 .
\end{aligned}
$$

Proposition 4.1. Under assumption (3.1) and if $\Phi_{1 \rho}$ satisfies (i), (ii), and (iii), problem $2^{\prime}$ has a solution.

Proof. For problem $2_{\varepsilon \rho n}^{\prime}$ we can prove, as in Proposition 3.1 by means of Brouwer's fixed-point theorem, that a solution exists and that

$$
\left\|\zeta_{i \varepsilon \rho n}\right\|_{Z_{i}} \leq c_{i}, \quad i=1,2,
$$

where $c_{i}, i=1,2$, are independent of $\varepsilon, \rho$, and $n$. Then as $\varepsilon \rightarrow 0, n \rightarrow \infty$,

$$
\zeta_{i \varepsilon \rho n} \rightarrow \zeta_{i \rho} \text { weakly in } Z_{i}, i=1,2 .
$$

Further, we can show as in Proposition 3.1 that (3.16) holds for $\left\{\beta_{\varepsilon}\left(\left[\zeta_{\varepsilon \rho}\right]\right\}\right.$. Thus

$$
\beta_{\varepsilon}\left(\left[\zeta_{\varepsilon \rho n}\right]\right) \rightarrow \chi_{\rho} \quad \text { weakly in } L^{1}\left(\Omega^{\prime}\right) .
$$

As $\rho \rightarrow 0$ we have

$$
\zeta_{i \rho} \rightarrow \zeta_{i} \text { weakly in } Z_{i}, i=1,2 \text {, }
$$

and

$$
\chi_{\rho} \rightarrow \chi \quad \text { weakly in } L^{1}\left(\Omega^{\prime}\right) .
$$

As in Proposition 3.1, we show that

$$
\left\|\operatorname{grad} \Phi_{1 \rho}\left(\zeta_{1 \varepsilon \rho n}\right)\right\|_{Z_{1}^{\prime}} \leq c
$$

where $c$ is independent of $\varepsilon, n, \rho$ and thus as $\varepsilon \rightarrow 0, n \rightarrow \infty$,

$$
\operatorname{grad} \Phi_{1 \rho}\left(\zeta_{1 \varepsilon \rho n}\right) \rightarrow \Psi_{\rho} \quad \text { weakly in } Z_{1}^{\prime}
$$

and

$$
\Psi_{\rho} \rightarrow \Psi \text { weakly in } Z_{1}^{\prime} .
$$

By means of the monotonicity argument for $\Phi$, we obtain as in Proposition 3.1

$$
\Psi_{\rho}=\operatorname{grad} \Phi_{\rho}\left(\zeta_{1 \rho}\right),
$$

where we use

$$
\lim _{\substack{\varepsilon \rightarrow 0 \\ n \rightarrow \infty}} \int_{\Omega^{\prime}} \beta_{\varepsilon}\left(\left[\zeta_{\varepsilon \rho n}\right]\right)\left[\zeta_{\varepsilon \rho n}\right] d \Omega=\int_{\Omega^{\prime}} \chi_{\rho}\left[\zeta_{\rho}\right] d \Omega
$$


The proof of (4.15) is the same as the proof of Lemma 3.1. By passing to the limits $\varepsilon \rightarrow 0, n \rightarrow \infty$ in (4.5) we obtain the variational equality

$$
\begin{aligned}
& \alpha_{1}\left(\zeta_{1 \rho}, z_{1}\right)+\alpha_{2}\left(\zeta_{2 \rho}, z_{2}\right)+\int_{\Omega^{\prime}} \chi_{\rho}[z] d \Omega+\left\langle\operatorname{grad} \Phi_{1 \rho}\left(\zeta_{1 \rho}\right), z_{1}\right\rangle \\
& =\left(\overline{\bar{f}}_{1}, z_{1}\right)+\left(\overline{\bar{f}}_{2}, z_{2}\right), \quad \forall z_{1} \in Z_{1}, \forall z_{2} \in Z_{2} .
\end{aligned}
$$

Now we pass to the limit $\rho \rightarrow 0$. From (4.16) and (4.14) we find that because of the convexity of $\Phi_{1 \rho}$

$$
\begin{aligned}
& \Phi_{1 \rho}\left(z_{1}\right)-\Phi_{1 \rho}\left(\zeta_{1 \rho}\right)+\alpha_{1}\left(\zeta_{1 \rho}, z_{1}-\zeta_{1 \rho}\right)+\alpha_{2}\left(\zeta_{2 \rho}, z_{2}-\zeta_{2 \rho}\right) \\
& \quad+\int_{\Omega^{\prime}} \chi_{\rho}\left([z]-\left[\zeta_{\rho}\right]\right) d \Omega \geq\left(\overline{\bar{f}}_{1}, z_{1}-\zeta_{1 \rho}\right)+\left(\overline{\bar{f}}_{2}, z_{2}-\zeta_{2 \rho}\right), \quad \forall z_{1} \in Z_{1}, \forall z_{2} \in Z_{2} .
\end{aligned}
$$

Let us introduce in (4.17) $z_{1}$ such that $\Phi_{1}\left(z_{1}\right)<\infty$. Then from (4.1) an $M_{1}$ exists such that $\Phi_{1 \rho}\left(z_{1}\right)<M_{1}$ and from (4.17) we obtain

$$
\Phi_{1 \rho}\left(\zeta_{1 \rho}\right) \leq M_{1}^{\prime}
$$

(4.18) and (4.9) imply (4.3). (4.17) is written in the form

$$
\begin{aligned}
A= & \Phi_{1 \rho}\left(z_{1}\right)+\alpha_{1}\left(\zeta_{1 \rho}, z_{1}\right)+\alpha_{2}\left(\zeta_{2 \rho}, z_{2}\right)+\int_{\Omega^{\prime}} \chi_{\rho}[z] d \Omega \\
\geq & \Phi_{1 \rho}\left(\zeta_{1 \rho}\right)+\alpha_{1}\left(\zeta_{1 \rho}, \zeta_{1 \rho}\right)+\alpha_{2}\left(\zeta_{2 \rho}, \zeta_{2 \rho}\right)+\int_{\Omega^{\prime}} \chi_{\rho}\left[\zeta_{\rho}\right] d \Omega \\
& +\left(\overline{\bar{f}}_{1}, z_{1}-\zeta_{1 \rho}\right)+\left(\overline{\bar{f}}_{2}, z_{2}-\zeta_{2 \rho}\right)=B, \quad \forall z_{1} \in Z_{1}, \forall z_{2} \in Z_{2},
\end{aligned}
$$

which as $\rho \rightarrow 0$ gives

$$
\begin{aligned}
\lim _{\rho \rightarrow 0} \inf A= & \lim _{\rho \rightarrow 0}\left\{\Phi_{1 \rho}\left(z_{1}\right)+\alpha_{1}\left(\zeta_{1 \rho}, z_{1}\right)+\alpha_{2}\left(\zeta_{2 \rho}, z_{2}\right)+\int_{\Omega^{\prime}} \chi_{\rho}[z] d \Omega\right\} \\
\geq & \lim _{\rho \rightarrow 0} \inf B=\lim _{\rho \rightarrow 0} \inf \left\{\Phi_{1 \rho}\left(\zeta_{1 \rho}\right)+\alpha_{1}\left(\zeta_{1 \rho}, \zeta_{1 \rho}\right)+\alpha_{2}\left(\zeta_{2 \rho}, \zeta_{2 \rho}\right)\right. \\
& \left.+\int_{\Omega^{\prime}} \chi_{\rho}\left[\zeta_{\rho}\right] d \Omega\right\}+\lim _{\rho \rightarrow 0}\left\{\left(\overline{\bar{f}}_{1}, z_{1}-\zeta_{1 \rho}\right)+\left(\overline{\bar{f}}_{2}, z_{2}-\zeta_{2 \rho}\right)\right\}, \\
& \forall z_{1} \in Z_{1}, \quad \forall z_{2} \in Z_{2} .
\end{aligned}
$$

From (4.3), (4.10), and the relation (proof as in Lemma 3.1)

$$
\lim _{\rho \rightarrow 0} \int_{\Omega^{\prime}} \chi_{\rho}\left[\zeta_{\rho}\right] d \Omega=\int_{\Omega^{\prime}} \chi[\zeta] d \Omega
$$

we get from (4.20) by means of

$$
\lim _{\rho \rightarrow 0} \inf \alpha_{i}\left(\zeta_{i \rho}, \zeta_{i \rho}\right) \geq \alpha_{i}\left(\zeta_{i}, \zeta_{i}\right), \quad i=1,2,
$$

the following:

$$
\begin{array}{r}
\Phi_{1}\left(z_{1}\right)-\Phi_{1}\left(\zeta_{1}\right)+\alpha_{1}\left(\zeta_{1}, z_{1}-\zeta_{1}\right)+\alpha_{2}\left(\zeta_{2}, z_{2}-\zeta_{2}\right)+\int_{\Omega^{\prime}} \chi([z]-[\zeta]) d \Omega \\
\geq\left(\overline{\bar{f}}_{1}, z_{1}-\zeta_{1}\right)+\left(\overline{\bar{f}}_{2}, z_{2}-\zeta_{2}\right), \quad \forall z_{1} \in Z_{1}, \quad \forall z_{2} \in Z_{2} .
\end{array}
$$

It remains for us to show that

$$
\chi \in \hat{\beta}([\zeta]) \text { a.e. on } \Omega^{\prime} \text {. }
$$

The proof of (4.24) is the same as in Proposition 3.1. 
5. On the strong convergence of the approximate solution. In Secs. 3 and 4 we have shown that $\zeta_{i \varepsilon n} \rightarrow \zeta_{i}$ weakly in $Z_{i}, i=1,2$. We shall study here the strong convergence of the solution by means of additional assumptions. We treat directly the nondifferentiable case.

Proposition 5.1. Suppose that there exist $q \geq 1$ and a constant $c>0$ such that

$$
|\beta(\xi)| \leq c\left(1+|\xi|^{q}\right) \quad \text { for all } \xi \in R .
$$

Moreover, let $b(\xi) \in \partial \varphi_{1}(\xi)$ and $b_{\rho}(\xi)=\operatorname{grad} \varphi_{1 \rho}(\xi)$ and let us assume that they satisfy the conditions

$$
|b(\xi)| \leq c_{1}(1+|\xi|)
$$

and

$$
\left|b_{\rho}(\xi)\right| \leq c_{2}(1+|\xi|)
$$

Then for $i=1,2$

$$
\zeta_{i \varepsilon n \rho} \rightarrow \zeta_{i} \quad \text { strongly in } Z_{i} \quad \text { as } \varepsilon \rightarrow 0, n \rightarrow \infty
$$

Proof. First we write (2.30) in the equivalent form

$$
\begin{aligned}
\alpha_{1}\left(\zeta_{1}, z_{1}-\zeta_{1}\right) & +\alpha_{2}\left(\zeta_{2}, z_{2}-\zeta_{2}\right)+\int_{\Omega^{\prime}} j^{0}([\zeta],[z]-[\zeta]) d \Omega \\
& +\tilde{\Phi}_{1}^{\prime}\left(\zeta_{1}, z_{1}-\zeta_{1}\right) \geq\left(\overline{\bar{f}}_{1}, z_{1}-\zeta_{1}\right)+\left(\overline{\bar{f}}_{2}, z_{2}-\zeta_{2}\right), \quad \forall z_{1} \in Z_{1}, \forall z_{2} \in Z_{2},
\end{aligned}
$$

where

$$
\tilde{\Phi}^{\prime}(\zeta, w)=\lim _{\lambda \rightarrow 0 .} \frac{\Phi(\zeta+\lambda w)-\Phi(\zeta)}{\lambda}
$$

is the one-sided directional Gâteaux differential at $\zeta$ in the direction $w$. It is well known that for $\Phi(\zeta)$ finite $\tilde{\Phi}(\zeta, \cdot)$ always exists [16]. To show the equivalence of (5.5) to (2.30) we note that setting in (2.30) $z_{i}-\zeta_{i}=\lambda\left(\tilde{z}_{i}-\zeta_{i}\right)$ for $0<\lambda<1$ implies (5.5). Conversely, (5.5) with the inequality

$$
\Phi_{1}\left(z_{1}\right)-\Phi_{1}\left(\zeta_{1}\right) \geq \Phi_{1}^{\prime}\left(\zeta_{1}, z_{1}-\zeta_{1}\right), \quad \forall z_{1} \in Z_{1}
$$

implies (2.30). Equation (5.5) implies by setting $z_{i}=\zeta_{i \varepsilon \rho n}, i=1,2$, that

$$
\begin{aligned}
\alpha_{1}\left(\zeta_{1}, \zeta_{1}\right)+\alpha_{2}\left(\zeta_{2}, \zeta_{2}\right) \leq & \alpha_{1}\left(\zeta_{1}, \zeta_{1 \varepsilon \rho n}\right)+\alpha_{2}\left(\zeta_{2}, \zeta_{2 \varepsilon \rho n}\right) \\
& +\int_{\Omega^{\prime}} j^{0}\left([\zeta],\left[\zeta_{\varepsilon \rho n}\right]-[\zeta]\right) d \Omega-\left(\overline{\bar{f}}_{1}, \zeta_{1 \varepsilon \rho n}-\zeta_{1}\right) \\
& -\left(\overline{\bar{f}}_{2}, \zeta_{2 \varepsilon \rho n}-\zeta_{2}\right)+\tilde{\Phi}_{1}^{\prime}\left(\zeta_{1}, \zeta_{1 \varepsilon \rho n}-\zeta_{1}\right) .
\end{aligned}
$$

From Problem $2_{\varepsilon \rho n}$ (Eq. (4.5)) we obtain

$$
\begin{aligned}
\alpha_{1}\left(\zeta_{1 \varepsilon, p n}, \zeta_{1 \varepsilon \rho n}\right)+ & \alpha_{2}\left(\zeta_{2 \varepsilon \rho n}, \zeta_{2 \varepsilon, p n}\right)=\alpha_{1}\left(\zeta_{1 \varepsilon \rho n}, z_{1 n}\right) \\
& +\alpha_{2}\left(\zeta_{2 \varepsilon, \rho n}, z_{2 n}\right)+\left\langle\operatorname{grad} \Phi_{1 \rho}\left(\zeta_{1 \varepsilon \rho n}\right), z_{1 n}-\zeta_{1 \varepsilon \rho n}\right\rangle \\
& +\int_{\Omega^{\prime}} \beta_{\varepsilon}\left(\left[\zeta_{\varepsilon, \rho n}\right]\right)\left(\left[z_{n}\right]-\left[\zeta_{\varepsilon, \rho n}\right]\right) d \Omega-\left(\overline{\bar{f}}_{1}, z_{1 n}-\zeta_{1 \varepsilon \rho n}\right) \\
& -\left(\overline{\bar{f}}_{2}, z_{2 n}-\zeta_{2 \varepsilon \rho n}\right), \quad \forall z_{1 n} \in Z_{1 n}, \forall z_{2 n} \in Z_{2 n} .
\end{aligned}
$$


Because of the coerciveness of the problem, we obtain from (5.8), (5.9)

$$
\begin{aligned}
c_{1} \| \zeta_{1} & -\zeta_{1 \varepsilon \rho n}\left\|^{2}+c_{2}\right\| \zeta_{2}-\zeta_{2 \varepsilon \rho n} \|^{2} \\
\leq & \alpha_{1}\left(\zeta_{1}-\zeta_{1 \varepsilon \rho n}, \zeta_{1}-\zeta_{1 \varepsilon \rho n}\right)+\alpha_{2}\left(\zeta_{2}-\zeta_{2 \varepsilon \rho n}, \zeta_{2}-\zeta_{2 \varepsilon \rho n}\right) \\
= & \alpha_{1}\left(\zeta_{1}, \zeta_{1}\right)+\alpha_{1}\left(\zeta_{1 \varepsilon \rho n}, \zeta_{1 \varepsilon \rho n}\right)+\alpha_{2}\left(\zeta_{2}, \zeta_{2}\right)+\alpha_{2}\left(\zeta_{2 \varepsilon \rho n}, \zeta_{2 \varepsilon \rho n}\right) \\
& -2 \alpha_{1}\left(\zeta_{1}, \zeta_{1 \varepsilon \rho n}\right)-2 \alpha_{2}\left(\zeta_{2}, \zeta_{2 \varepsilon \rho n}\right) \\
\leq & \alpha_{1}\left(\zeta_{1}, \zeta_{1 \varepsilon \rho n}\right)+\alpha_{2}\left(\zeta_{2}, \zeta_{2 \varepsilon \rho n}\right)+\int_{\Omega^{\prime}} j^{0}\left([\zeta],\left[\zeta_{\varepsilon \rho n}\right]-[\zeta]\right) d \Omega \\
& -\left(\overline{\bar{f}}_{1}, \zeta_{1 \varepsilon \rho n}-\zeta_{1}\right)-\left(\overline{\bar{f}}_{2}, \zeta_{2 \varepsilon \rho n}-\zeta_{2}\right)+\tilde{\Phi}_{1}^{\prime}\left(\zeta_{1}, \zeta_{1 \varepsilon \rho n}-\zeta_{1}\right) \\
& +\alpha_{1}\left(\zeta_{1 \varepsilon \rho n}, z_{1 n}\right)+\alpha_{2}\left(\zeta_{2 \varepsilon \rho n}, z_{2 n}\right)+\left\langle\operatorname{grad} \Phi_{1 \rho}\left(\zeta_{1 \varepsilon \rho n}\right), z_{1 n}-\zeta_{1 \varepsilon \rho n}\right\rangle \\
& +\int_{\Omega^{\prime}} \beta_{\varepsilon}\left(\left[\zeta_{\varepsilon \rho n}\right]\right)\left(\left[z_{n}\right]-\left[\zeta_{\varepsilon \rho n}\right]\right) d \Omega-\left(\overline{\bar{f}}_{1}, z_{1 n}-\zeta_{1 \varepsilon \rho n}\right) \\
& -\left(\overline{\bar{f}}_{2}, z_{2 n}-\zeta_{2 \varepsilon \rho n}\right)-2 \alpha_{1}\left(\zeta_{1}, \zeta_{1 \varepsilon \rho n}\right)-2 \alpha_{2}\left(\zeta_{2}, \zeta_{2 \varepsilon \rho n}\right), \quad \forall z_{1 n} \in Z_{1 n}, \forall z_{2 n} \in Z_{2 n} .
\end{aligned}
$$

We take $z_{\text {in }}, i=1,2$, such that as $n \rightarrow \infty$

$$
z_{\text {in }} \rightarrow \zeta_{i} \text { strongly in } Z_{i} \subset H^{2}\left(\Omega_{i}\right), i=1,2 .
$$

From (5.10) by considering the limit $\varepsilon \rightarrow 0, n \rightarrow \infty, \rho \rightarrow 0$ we have to calculate

$$
\begin{aligned}
\lim \left\{\int _ { \Omega ^ { \prime } } j ^ { 0 } \left([\zeta],\left[\zeta_{\varepsilon \rho n}\right]\right.\right. & -[\zeta]) d \Omega+\int_{\Omega^{\prime}} \beta_{\varepsilon}\left(\left[\zeta_{\varepsilon \rho n}\right]\right)\left(\left[z_{n}\right]-\left[\zeta_{\varepsilon \rho n}\right]\right) d \Omega \\
& \left.+\tilde{\Phi}_{1}^{\prime}\left(\zeta_{1}, \zeta_{1 \varepsilon \rho n}-\zeta_{1}\right)+\left\langle\operatorname{grad} \Phi_{1 \rho}\left(\zeta_{1 \varepsilon \rho n}\right), z_{1 n}-\zeta_{1 \varepsilon \rho n}\right\rangle\right\}
\end{aligned}
$$

Using hypothesis (5.1) and the definition of $j^{0}$, we have

$$
\begin{aligned}
& \int_{\Omega^{\prime}} j^{0}\left([\zeta],\left[\zeta_{\varepsilon \rho n}\right]-[\zeta]\right) d \Omega=\int_{\Omega^{\prime}} \lim _{\substack{\lambda \rightarrow 0_{+} \\
h \rightarrow 0}} \sup \frac{1}{\lambda} \int_{[\zeta]+h}^{[\zeta]+h+\lambda\left\{\left[\zeta_{c, m}\right]-[\zeta]\right\}} \beta(t) d t d \Omega \\
& \leq c \int_{\Omega^{\prime}} \lim _{\substack{\lambda \rightarrow 0_{+} \\
h \rightarrow 0}} \sup \frac{1}{\lambda} \int_{[\zeta]+h}^{[\zeta]+h+\lambda\left\{\left[\zeta_{c m}\right]-[\zeta]\right\}}\left(1+|t|^{q}\right) d t d \Omega \\
& =c \int_{\Omega^{\prime}}\left(1+[\zeta]^{q}\right)\left(\left[\zeta_{\varepsilon \rho n}\right]-[\zeta]\right) d \Omega .
\end{aligned}
$$

Since $H^{2}\left(\Omega_{i}\right)$ is compactly imbedded into $L^{q}\left(\Omega_{i}\right), q \geq 1, i=1$, we obtain, since $\Omega^{\prime} \subset \Omega_{i}$ and using Minkowski's inequality, $\left[\zeta_{\varepsilon \rho n}\right] \rightarrow[\zeta]$ strongly in $L^{q}\left(\Omega^{\prime}\right), q \geq 1$. Thus for $\varepsilon \rightarrow 0, n \rightarrow \infty, \rho \rightarrow 0$

$$
\lim \int_{\Omega^{\prime}} j^{0}\left([\zeta],\left[\zeta_{\varepsilon \rho n}\right]-[\zeta]\right) d \Omega=0
$$

From (4.8), (4.10)

$$
\left\|\beta_{\varepsilon}\left(\left[\zeta_{\varepsilon \rho n}\right]\right)\right\|_{L^{\prime}\left(\Omega^{\prime}\right)}<c
$$

Moreover, the compact imbedding $H^{2}\left(\Omega_{i}\right) \subset C^{0}\left(\bar{\Omega}_{i}\right), i=1,2$, implies that

$$
\left[\zeta_{\varepsilon p n}\right] \rightarrow[\zeta] \text { strongly in } L^{\infty}\left(\Omega^{\prime}\right) \text {. }
$$


Therefore, we have that

$$
\begin{aligned}
\mid \int_{\Omega^{\prime}} & \beta_{\varepsilon}\left(\left[\zeta_{\varepsilon \rho n}\right]\right)\left(\left[z_{n}\right]-\left[\zeta_{\varepsilon \rho n}\right]\right) d \Omega \mid \\
& \leq\left\|\beta_{\varepsilon}\left(\left[\zeta_{\varepsilon \rho n}\right]\right)\right\|_{L^{\prime}\left(\Omega^{\prime}\right)}\left\|\left[z_{n}\right]-\left[\zeta_{\varepsilon \rho n}\right]\right\|_{L^{\infty}\left(\Omega^{\prime}\right)} \\
& \leq c\left\|\left[z_{n}\right]-\left[\zeta_{\varepsilon \rho n}\right]\right\|_{L^{\infty}\left(\Omega^{\prime}\right)} \\
& \leq c\left\|\left[z_{n}\right]-[\zeta]\right\|_{L^{\infty}\left(\Omega^{\prime}\right)}+c\left\|[\zeta]-\left[\zeta_{\varepsilon \rho}\right]\right\|_{L^{\infty}\left(\Omega^{\prime}\right) .}
\end{aligned}
$$

From this inequality we obtain as $\varepsilon \rightarrow 0, n \rightarrow \infty, \rho \rightarrow 0$

$$
\lim \int_{\Omega^{\prime}} \beta_{\varepsilon}\left(\left[\zeta_{\varepsilon \rho n}\right]\right)\left(\left[z_{n}\right]-\left[\zeta_{\varepsilon \rho n}\right]\right) d \Omega=0 .
$$

We examine the term $\tilde{\Phi}_{1}^{\prime}\left(\zeta_{1}, \zeta_{1 \varepsilon \rho n}-\zeta_{1}\right)$. From assumption (5.2) we easily obtain $\varphi_{1}(\partial \zeta / \partial n) \in L^{1}(\Gamma)$, by applying the trace theorem and using the fact that up to an additive constant $\varphi_{1}(\xi)=\int_{\xi_{0}}^{\xi} b(\xi) d \xi\left(b\left(\xi_{0}\right)\right.$ finite), where $b$ is a measurable selection of $\hat{b}$. Moreover, $\Phi_{1}$ is regular in the sense of Clarke and therefore $\Phi_{1}^{0}(w, z)=\tilde{\Phi}_{1}^{\prime}(w, z)$. Using assumption (5.2) and the continuity of the trace mapping, we obtain by applying Proposition 2.3.6 of [7] (see also [3], Proposition 4.3.1)

$$
\Phi_{1}^{\prime}(w, z)=\Phi_{1}^{0}(w, z)=\int_{\Gamma} \varphi_{1}^{0}\left(\frac{\partial w}{\partial n}, \frac{\partial z}{\partial n}\right) d \Gamma=\int_{\Gamma} \tilde{\varphi}_{1}^{\prime}\left(\frac{\partial w}{\partial n}, \frac{\partial z}{\partial n}\right) d \Gamma .
$$

But

$$
\begin{aligned}
& \int_{\Gamma} \tilde{\varphi}_{1}^{\prime}\left(\frac{\partial w}{\partial n}, \frac{\partial z}{\partial n}\right) d \Gamma=\int_{\Gamma} \lim _{\lambda \rightarrow 0_{+}} \frac{1}{\lambda} \int_{\partial w / \partial n}^{\partial w / \partial n+\lambda \partial z / \partial n} b(t) d t \\
& \quad \leq \int_{\Gamma^{\lambda} \rightarrow 0_{+}} \frac{1}{\lambda} \int_{\partial w / \partial n}^{\partial w / \partial n+\lambda \partial z / \partial n}(1+|t|) d t=c \int_{\Gamma}\left(1+\left|\frac{\partial w}{\partial n}\right|\right)\left(\frac{\partial z}{\partial n}\right) d \Gamma \\
& \quad \leq c\left\|1+\frac{\partial w}{\partial n}\right\|_{L^{2}(\Gamma)}\left\|\frac{\partial z}{\partial n}\right\|_{L^{2}(\Gamma)} .
\end{aligned}
$$

We note that $\zeta_{1 \varepsilon \rho n} \rightarrow \zeta_{1}$ weakly in $H^{2}\left(\Omega_{1}\right)$ implies that $\partial \zeta_{1 \varepsilon \rho n} / \partial n \rightarrow \partial \zeta_{1} / \partial n$ strongly in $L^{2}\left(\Gamma_{1}\right)$ for a Lipschitzian boundary $\Gamma_{1}$. Indeed, $\zeta_{1 \varepsilon \rho n} \rightarrow \zeta_{1}$ weakly in $H^{2}\left(\Omega_{1}\right)$ implies that $\zeta_{1 \varepsilon \rho n, i} \rightarrow \zeta_{1, i}$ weakly in $H^{1}\left(\Omega_{1}\right)$ and, because $\left.u \in H^{1}\left(\Omega_{1}\right) \rightarrow u\right|_{\Gamma} \in L^{2}\left(\Gamma_{1}\right)$ is compact and $n=\left\{n_{j}\right\}$ exists a.e. on $\Gamma_{1}$ where $n_{j}$ is a measurable bounded function, we get the strong convergence of the normal derivatives on $\Gamma_{1}$. Thus as $\varepsilon \rightarrow 0, \rho \rightarrow 0$, $n \rightarrow \infty$

$$
\lim \tilde{\Phi}_{1}^{\prime}\left(\zeta_{1}, \zeta_{1 \varepsilon \rho n}-\zeta_{1}\right)=0
$$

We now examine the term $\left\langle\operatorname{grad} \Phi_{1 \rho}\left(\zeta_{1 \varepsilon \rho n}\right), z_{1 n}-\zeta_{1 \varepsilon \rho n}\right\rangle$. Using the same arguments as in the previous case, we find using (5.3) that

$$
\begin{aligned}
\left\langle\operatorname{grad} \Phi_{1 \rho}(w), z\right\rangle & =\int_{\Gamma^{\prime}} \operatorname{grad} \varphi_{1 \rho}\left(\frac{\partial w}{\partial n}\right) \frac{\partial z}{\partial n} d \Gamma \\
& =\int_{\Gamma^{\prime}} \lim _{\lambda \rightarrow 0_{+}} \frac{1}{\lambda} \int_{\partial^{w} / \partial n}^{\partial w / \partial n+\lambda \partial z / \partial n} b_{\rho}(t) d t \leq c \int_{\Gamma}\left(1+\left|\frac{\partial w}{\partial n}\right|\right)\left(\frac{\partial z}{\partial n}\right) d \Gamma \\
& \leq c\left\|1+\left|\frac{\partial w}{\partial n}\right|\right\|_{L^{2}(\Gamma)}\left\|\frac{\partial z}{\partial n}\right\|_{L^{2}(\Gamma)}
\end{aligned}
$$


Thus

$$
\begin{aligned}
\mid\langle\operatorname{grad} & \left.\Phi_{1 \rho}\left(\zeta_{1 \varepsilon \rho n}\right), z_{1 n}-\zeta_{1 \varepsilon \rho n}\right\rangle \\
& \leq c\left\|1+\left|\frac{\partial \zeta_{1 \varepsilon \rho n}}{\partial n}\right|\right\|_{L^{2}(\Gamma)}\left\|\frac{\partial z_{1 n}}{\partial n}-\frac{\partial \zeta_{1 \varepsilon \rho n}}{\partial n}\right\|_{L^{2}(\Gamma)} \\
& \leq c\left\|\frac{\partial z_{1 n}}{\partial n}-\frac{\partial \zeta_{1}}{\partial n}\right\|_{L^{2}(\Gamma)}+c\left\|\frac{\partial \zeta_{1}}{\partial n}-\frac{\partial \zeta_{1 \varepsilon \rho n}}{\partial n}\right\|_{L^{2}(\Gamma)}
\end{aligned}
$$

which implies that as $\varepsilon \rightarrow 0, \rho \rightarrow 0, n \rightarrow \infty$

$$
\lim \left\langle\operatorname{grad} \Phi_{1 \rho}\left(\zeta_{1 \varepsilon \rho n}\right), z_{1 n}-\zeta_{1 \varepsilon \rho n}\right\rangle=0
$$

Thus (5.12) tends to zero, and this implies with (5.10) the strong convergence of $\zeta_{i \varepsilon \rho n}$ to $\zeta_{i}$ in $H^{2}\left(\Omega_{i}\right)$ for $i=1,2$.

Because of this strong convergence we will also have convergence of $\zeta_{i \varepsilon \rho n}$ to $\zeta_{i}$ in the $C^{0}\left(\bar{\Omega}_{i}\right)$-norm.

6. Related general boundary-value problems and remarks. Analogous results may be obtained if other types of subdifferential boundary conditions hold. Suppose for instance that on $\Gamma_{1}^{\prime} \subset \Gamma_{1}$ (2.20) holds and that the remaining boundary conditions on $\Gamma_{1}$ and $\Gamma_{2}$ guarantee the coerciveness of the bilinear forms. We denote by $Z_{i}, i=1,2$, the closed subspaces of $H^{2}\left(\Omega_{i}\right), i=1,2$, defining the kinematically admissible sets of the plates. The resulting variational-hemivariational inequality is analogous to (2.30) but with $\Phi_{1}$ replaced by $\Phi_{1}^{\prime}$, which is given by (2.26). Again Propositions 3.1 and 4.1 hold and the problem admits a solution. The proof of Proposition 5.1 is slightly modified since the imbedding $H^{2}(\Omega) \rightarrow L^{\infty}(\Gamma)$ is compact. Moreover, assumptions (5.2) and (5.3) can be replaced by the assumptions

$$
\left|b^{\prime}(\xi)\right| \leq c_{1}\left(1+|\xi|^{q_{1}}\right), \quad q_{1} \geq 1
$$

and

$$
\left|b_{\rho}^{\prime}(\xi)\right| \leq c_{2}\left(1+|\xi|^{q_{2}}\right), \quad q_{2} \geq 1,
$$

where $b^{\prime}(\xi) \in \partial \varphi_{1}^{\prime}(\xi)$ and $b_{\rho}^{\prime}(\xi)=\operatorname{grad} \varphi_{1 \rho}^{\prime}(\xi)$. Moreover, instead of (6.2) we may assume that

$$
\xi \rightarrow b_{\rho}^{\prime}(\xi) \in C^{0}(R) .
$$

Also, combinations of subdifferential boundary conditions can be dealt with. Suppose, e.g., that on $\Gamma_{i}^{\prime} \subset \Gamma_{i}$ and $\Gamma_{i}^{\prime \prime} \subset \Gamma_{i}$ the plates $\Omega_{i}$ are subjected to the boundary conditions $^{1}$ (2.19) and (2.21) on $\Gamma_{i}^{\prime}$ and (2.20) and (2.22) on $\Gamma_{i}^{\prime \prime}$, respectively, and that on the remaining parts $\Gamma_{i}^{\prime \prime \prime}$ of their boundaries they are subjected to classical

\footnotetext{
${ }^{1}$ Various types of boundary conditions may simplify some parts of the proof. (The proof of Problem 2 is the most general possible.) For instance, due to the imbeddings $H^{2}(\Omega) \subset C^{0}(\bar{\Omega}) \subset C^{0}(\Gamma) \subset L^{\infty}(\Gamma)$ and the smoothness of $\beta_{\varepsilon}$ the part of the proof (3.16-3.23) is superfluous for the variational-hemivariational inequality (2.30). However, the $L^{1}$-weak precompactness of $\left\{\beta_{\varepsilon}\left(\zeta_{\varepsilon \eta}\right)\right\}$ has to be shown in any problem for which the previous imbeddings do not hold, e.g., for a hemivariational inequality formulated in the $H^{1}$-space (see [3], p. 272). This is also the case in our problem if a nonmonotone boundary condition holds, i.e., if (2.19) is replaced by
}

$$
M \in \hat{b}_{1}\left(\frac{\partial \zeta_{1}}{\partial n}\right)=\bar{\partial} \varphi_{1}\left(\frac{\partial \zeta_{1}}{\partial n}\right) \text { on } \Gamma_{1}
$$


boundary conditions which guarantee the coerciveness of the bilinear forms. Then we may pose the problem in the general form (2.27). We may also define, for $i=1,2$, the convex, l.s.c., and proper functionals

$$
\bar{\varphi}_{i}\left(z_{i}\right)= \begin{cases}\int_{\Gamma_{1}^{\prime}} \varphi_{i}\left(\frac{\partial z_{i}}{\partial n}\right) d \Gamma+\int_{\Gamma_{\prime}^{\prime \prime}} \varphi_{i}^{\prime}\left(z_{i}\right) d \Gamma & \text { if } \varphi_{i}\left(\frac{\partial z_{i}}{\partial n}\right) \in L^{1}\left(\Gamma_{i}^{\prime}\right) \\ \infty \text { otherwise } & \text { and } \varphi_{i}^{\prime}\left(z_{i}\right) \in L^{1}\left(\Gamma_{i}^{\prime \prime}\right),\end{cases}
$$

and pose the problem again in a form similar to (2.27) but now including the terms

$$
\sum_{i=1}^{2}\left(\bar{\Phi}_{i}\left(z_{i}\right)-\bar{\Phi}_{i}\left(\zeta_{i}\right)\right) \quad \text { given by (6.3) }
$$

To study this problem we make the same assumptions as in Propositions 3.1 and 4.1 but now for each $\bar{\Phi}_{i}$. The same proofs (with only slight modifications) as in Proposition 3.1 and Proposition 4.1 yield the existence of the solution for both the differentiable and the nondifferentiable case. The strong convergence is shown under the growth assumptions (5.1) and (5.2), (5.3) where now the latter have to be assumed for the $b_{i}^{\prime}$ s corresponding to $\varphi_{i}$ and $\varphi_{i}^{\prime}, i=1,2$. As noticed before, the growth assumptions with respect to the $\varphi_{i}^{\prime}$ s may be replaced by (6.1) and (6.2) whereas the latter may also be replaced by $\xi \rightarrow b_{i \rho}^{\prime}(\zeta) \in C^{0}(R)$.

We may also obtain existence and approximation results if subdifferential conditions hold in $\Omega_{i}^{\prime \prime} \subset \Omega_{i}, i=1,2$, of the following form. Suppose that

$$
\begin{aligned}
\overline{\overline{f_{i}}} & =\overline{\overline{f_{i}^{\prime}}}+\overline{\overline{f_{i}^{\prime \prime}}}, \quad \text { where } \overline{\overline{f_{i}^{\prime}}} \text { is given in } L^{2}\left(\Omega_{i}\right) \text { and } \\
-\overline{\overline{f_{i}^{\prime \prime}}} & \in \partial \varphi_{i}^{\prime \prime}\left(\zeta_{i}\right) \text { on } \Omega_{i}^{\prime \prime} \subset \Omega_{i}, \\
\overline{\overline{f_{i}^{\prime \prime}}} & =0 \text { on } \Omega_{i}-\Omega_{i}^{\prime \prime},
\end{aligned}
$$

where $\bar{\Omega}_{i} \cap \Gamma_{i}=\varnothing$. Here $\varphi_{i}^{\prime \prime}$ is a convex, 1.s.c., proper functional on R. It is well known (cf. [3], p. 91) that (6.4) describes the unilateral contact problem for each plate (cf. Fig. 3) for a deformable or a rigid support. In this case $\Phi_{i}\left(z_{i}\right)$ in (6.3) will also contain the term $\left\{\int_{\Omega_{1}^{\prime \prime}} \varphi_{i}^{\prime \prime}\left(\zeta_{i}\right) d \Omega\right.$ if $\varphi_{i}^{\prime \prime}\left(\zeta_{i}\right) \in L^{1}(\Omega), \infty$ otherwise $\}$. Again Propositions 3.1 and 4.1 hold under the same assumptions as before, and analogously strong convergence may also be shown. In this case $b_{i}^{\prime \prime}(\xi) \in \partial \varphi_{i}^{\prime \prime}(\xi)$ has to satisfy a relation of the form $(6.1)$ and $b_{\rho i}^{\prime \prime}(\xi)=\operatorname{grad} \varphi_{\rho i}^{\prime \prime}(\xi)$ a relation analogous to (6.2) or that $\xi \rightarrow b_{\rho i}^{\prime \prime}(\xi) \in C^{0}(R)$. The method developed can also be applied to orthotropic or anisotropic plates by changing appropriately the bilinear forms $\alpha_{i}\left(\zeta_{i}, z_{i}\right)$.

Until now we have considered a laminated plate consisting of two laminae. The same results can be obtained for a laminated plate consisting of $n$ laminae which

Then we take the Galerkin approximation

$$
Z_{1 n} \subset \bar{Z}_{1}=Z_{1} \cap\left\{z_{1}\left|z_{1} \in Z_{1} \cdot \frac{\partial z_{1}}{\partial n}\right|_{\Gamma_{1}} \in L^{\infty}\left(\Gamma_{1}\right)\right\}
$$

where $\hat{Z}_{1}$ is dense in $Z_{1} ;(3.14)$ is replaced by $i \zeta_{1: n} / \partial n \rightarrow \partial \zeta_{1} / \partial n$ strongly in $L^{2}\left(\Gamma_{1}\right)$ and then the $L^{1}$-weak precompactness of $\left\{h_{1 \varepsilon}\left(\partial \zeta_{1: n} / \partial n\right)\right\}$ has to be proved (cf.(3.19-3.24)). 
are bonded by interface laws of the nonmonotone possibly multivalued form (2.4)(2.6). Let us assume that all the subdifferential boundary conditions of each plate are included in a functional $\Phi_{i}$ defined as in (6.3), whereas the classical boundary conditions are taken into account in the definition of the subspaces $Z_{i}, i=1,2, \ldots, n$. Then we may formulate the following problem.

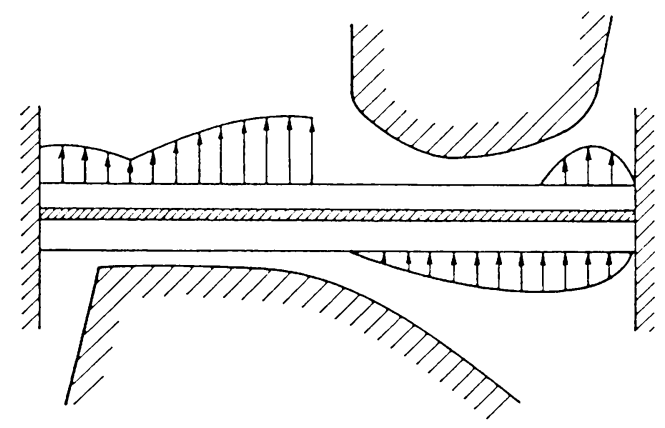

FIG. 3. On the obstacle problem for a laminated plate

Problem 3. Find $\zeta_{i} \in Z_{i}, i=1,2, \ldots, n$, such as to satisfy the variationalhemivariational inequality

$$
\begin{aligned}
\sum_{i=1}^{n} \alpha_{i}\left(\zeta_{i}, z_{i}-\zeta_{i}\right) & +\sum_{i=1}^{n-1} \int_{\Omega_{i}^{\prime}} j_{i}^{0}\left([\zeta]_{i},[z]_{i}-[\zeta]_{i}\right) d \Omega^{\prime} \\
& +\sum_{i=1}^{n}\left(\Phi_{i}\left(z_{i}\right)-\Phi_{i}\left(\zeta_{i}\right)\right) \geq \sum_{i=1}^{n} \int_{\Omega_{i}} \overline{\bar{f}}_{i}\left(z_{i}-\zeta_{i}\right) d \Omega, \quad \forall z_{i} \in Z_{i} .
\end{aligned}
$$

Here $[z]_{i}=z_{i+1}-z_{i}, i=1,2, \ldots, n-1$.

Following the same methods as in Propositions 3.1, 4.1, 5.1 we can prove the following proposition on the assumption that the boundary conditions guarantee the coerciveness of the bilinear forms. (For example, let us assume for simplicity that on $\Gamma_{i}^{\prime} \subset \Gamma_{i}$ each plate is clamped, i.e.,that (2.28) holds for each plate.) Moreover, we assume that $\overline{\bar{f}}_{i} \in L^{2}\left(\Omega_{i}\right)$.

Proposition 6.1. Let (3.2) hold for the $\beta_{i}$ corresponding to $j_{i}, i=1,2, \ldots, n$, and assume that for each $\Phi_{i}, i=1,2, \ldots, n$, assumptions (4.1)-(4.3) hold. Then problem 3 admits a solution.

The proof is the same as the proof of Propositions 3.1 and 4.1 and therefore is omitted here. Analogously, we obtain the strong convergence and therefore the convergence in the $C^{0}\left(\bar{\Omega}_{i}\right)$-norm, if growth assumptions (5.2), (5.3) and/or (6.1), (6.2) hold.

It is worth noting that by applying the method of [3], p. 150 all the studied problems can be formulated as substationarity problems of the potential energy. Moreover, because of the lack of convexity the problem does not have a unique solution. Each local minimum of the potential energy is a substationarity point and therefore a solution of the variational-hemivariational inequality-and therefore of the problem-but not conversely. 
The method applied to the study of the existence and approximation of the solution of laminated plates in Propositions 3.1 and 4.1 can also be applied to the study of the existence and approximation of the solution of more abstract mathematical problems. We mean here nonmonotone perturbations (expressed in terms of generalized gradients) of multivalued equations of the form $f-A u \in \partial \Phi(u)$, i.e., the study of multivalued equations of the form

$$
f-A u \in \partial \Phi(u)+\bar{\partial} \zeta(u) .
$$

Here $\bar{\partial} \zeta$ introduces the nonmonotone perturbations of the equation, $\Phi$ is a convex, l.s.c., and proper functional on a Banach space $V, f \in V^{\prime}$, and $A: V \rightarrow V^{\prime}$ is a linear, bounded coercive operator.

\section{REFERENCES}

[1] P. D. Panagiotopoulos, Nonconvex superpotentials in the sense of F. H. Clarke and applications, Mech. Res. Comm. 8, 335-340 (1981)

[2] P. D. Panagiotopoulos, Nonconvex energy functions. Hemivariational irequalities and substationarity principles, Acta Mech. 48, 160-183 (1983)

[3] P. D. Panagiotopoulos, Inequality problems in mechanics and applications. Convex and non-convex energy functions, Birkhäuser Verlag, Basel, Boston, Stuttgard, 1985

[4] J. J. Moreau, La notion de sur-potentiel et les liaisons unilatérales en élastostatique, C. R. Acad. Sci. Paris Ser. A 267, 954-957 (1968)

[5] P. D. Panagiotopoulos, Ioffe fans and unilateral problems: a new conjecture, Proc. 3rd CISM Meeting on Unilateral Problems in Structural Analysis, Prescudin Udine, June 1985, CISM Courses and Lectures, Vol. 304, Springer-Verlag, Wien, New York, pp. 239-257, 1987

[6] G. Duvaut and J. L. Lions, Les inéquations en méchanique et en physique, Dunod, Paris, 1972 (English translation: Inequalities in mechanics and physics, Springer-Verlag, Berlin, Heidelberg, New York, 1976)

[7] F. H. Clarke, Nonsmooth analysis and optimization, Wiley, New York, 1984

[8] P. D. Panagiotopoulos, Une généralisation non-convexe de la notion du sur-potentiel et ses applications, C. R. Acad. Sci. Paris Sér. B 296, 580-584 (1983)

[9] P. D. Panagiotopoulos, Hemivariational inequalities and substationarity in the static theory of $v$. Kármán plates, Z. Angew. Math. Mech. 65, 219-229 (1985)

[10] R. Jones, Mechanics of composite materials, McGraw-Hill-Scripta, New York, Washington, D. C., 1975

[11] K. C. Chang, Variational methods for non-differentiable functionals and their applications to partial differential equations, J. Math. Anal. Appl. 80, 102-129 (1981)

[12] H. Brezis, Problèmes unilatéraux, J. Math. Pures Appl. 51, 1-168 (1972)

[13] J. J. Moreau, Fonctionnelles convexes, Séminaire au Collége de France, 1966-1967

[14] J. Ekeland and R. Temam, Convex analysis and variational problems, North-Holland, Amsterdam, and American Elsevier, New York, 1976

[15] J. Rauch, Discontinuous semilinear differential equations and multiple valued maps, Proc. Amer. Math. Soc. 64, 277-282 (1977)

[16] R. T. Rockafellar, Convex analysis, Princeton University Press, Princeton, N. J., 1970

[17] J. P. Aubin and F. H. Clarke, Shadow prices and duality for a class of optimal control problems, SIAM J. Control Optim. 17, 567-586 (1979) 